\title{
Circulating protein synthesis rates reveal skeletal muscle proteome dynamics
}

\author{
Mahalakshmi Shankaran, ${ }^{1}$ Chelsea L. King, ${ }^{1}$ Thomas E. Angel, ${ }^{1}$ William E. Holmes, ${ }^{1}$ Kelvin W. Li, ${ }^{1}$ Marc Colangelo, ${ }^{1}$ John C. Price, ${ }^{1}$ \\ Scott M. Turner, ${ }^{1}$ Christopher Bell, ${ }^{2}$ Karyn L. Hamilton, ${ }^{2}$ Benjamin F. Miller, ${ }^{2}$ and Marc K. Hellerstein ${ }^{1,3}$ \\ 1KineMed Inc., Emeryville, California, USA. ²Department of Health and Exercise Science, Colorado State University, Fort Collins, Colorado, USA. ${ }^{3}$ Department of Nutritional Sciences, University of California, \\ Berkeley, California, USA
}

\begin{abstract}
Here, we have described and validated a strategy for monitoring skeletal muscle protein synthesis rates in rodents and humans over days or weeks from blood samples. We based this approach on label incorporation into proteins that are synthesized specifically in skeletal muscle and escape into the circulation. Heavy water labeling combined with sensitive tandem mass spectrometric analysis allowed integrated synthesis rates of proteins in muscle tissue across the proteome to be measured over several weeks. Fractional synthesis rate (FSR) of plasma creatine kinase M-type (CK-M) and carbonic anhydrase 3 (CA-3) in the blood, more than $90 \%$ of which is derived from skeletal muscle, correlated closely with FSR of CK-M, CA-3, and other proteins of various ontologies in skeletal muscle tissue in both rodents and humans. Protein synthesis rates across the muscle proteome generally changed in a coordinate manner in response to a sprint interval exercise training regimen in humans and to denervation or clenbuterol treatment in rodents. FSR of plasma CK-M and CA-3 revealed changes and interindividual differences in muscle tissue proteome dynamics. In human subjects, sprint interval training primarily stimulated synthesis of structural and glycolytic proteins. Together, our results indicate that this approach provides a virtual biopsy, sensitively revealing individualized changes in proteome-wide synthesis rates in skeletal muscle without a muscle biopsy. Accordingly, this approach has potential applications for the diagnosis, management, and treatment of muscle disorders.
\end{abstract}

\section{Introduction}

Disorders of muscle mass, quality, and function cause substantial and increasing morbidity and mortality. Sarcopenia, cachexia, and frailty are growing in importance in association with aging demographics world-wide $(1,2)$. Loss of skeletal muscle mass is a major target for drug development (3), but this field has been held back by the absence of simple translational biomarkers that can be used for diagnosis, prognosis, and monitoring. The synthesis and breakdown rates of skeletal muscle proteins of different classes are perturbed in muscle-wasting conditions (4) and increasing muscle protein synthesis is the primary metabolic mechanism of action of anabolic interventions proven to increase muscle mass and strength, such as resistance exercise and androgen treatment (5-10). Changes in mixed muscle protein synthesis rates occur very rapidly in response to anabolic interventions in humans $(8,11)$ - in advance of changes in muscle mass, strength, or performance $(5,9,10)$ - and therefore would be ideal biomarkers for early assessment prediction and monitoring of treatment efficacy.

Conflict of interest: M. Shankaran, C.L. King, T.E. Angel, W.E. Holmes, K.W. Li, M. Colangelo, and S.M. Turner are employees of KineMed Inc. M.K. Hellerstein is the chief of the scientific and clinical advisory boards of KineMed Inc. J.C. Price is a former employee of KineMed Inc. M. Shankaran, C.L. King, T.E. Angel, W.E. Holmes, K.W. Li, M. Colangelo, J.C. Price, S.M. Turner, and M.K. Hellerstein own stock in the company. Submitted: October 22, 2014; Accepted: November 5, 2015 Reference information: / Clin Invest. 2016;126(1):288-302. doi:10.1172/JCI79639.
Translatable metrics of skeletal muscle protein turnover that can be applied routinely in therapeutic trials or in the clinic have, however, not been available. Blood- or urine-based tests of intracellular protein turnover in skeletal muscle would be particularly useful as biomarkers. An accurate, minimally invasive test of muscle protein dynamics might have applications for early detection of therapeutic response to therapeutic interventions, patient selection, and translating results from animal models to humans.

Fractional synthesis rates (FSRs) of mixed muscle proteins or protein subfractions are typically measured in rodents and humans through short-term infusions of stable isotope-labeled amino acids (12-16). These methods have demonstrated the anabolic effects of exercise $(6,7)$, dietary supplements $(17,18)$, or treatment with anabolic agents such as testosterone (8-10) or clenbuterol (19, 20). Although changes in FSR measurements precede longer-term responses of muscle mass and strength $(5,8-10)$, measurement of acute synthesis rates of mixed proteins in muscle has a number of fundamental limitations. First, the integrated effects on protein turnover of diet, activity, hormones, and medications over days or weeks are more relevant to muscle mass and function than turnover rates over hours, particularly for the long-lived structural and mitochondrial proteins that are characteristic of skeletal muscle. Second, broad interrogation of dynamics across proteins in different classes within the proteome is required to explore the coordinated control of expression and catabolism of different functional classes of proteins or to identify protein turnover signatures of diseases or interventions. And, third, measurement of muscle protein 
kinetics has previously required a tissue sample rather than being measurable noninvasively through a body fluid measurement.

A solution to the first problem is the use of oral intake of heavy water $\left({ }^{2} \mathrm{H}_{2} \mathrm{O}\right)$ in the outpatient setting to label newly synthesized proteins over periods of days, weeks, or months (21-28). We $(25,29-31)$ and others $(32,33)$ have shown that the second problem can be addressed by combining stable isotope label incorporation with tandem mass spectrometric-based proteomics techniques. In particular, isotope ratio measurements using liquid chromatography-mass spectrometry/mass spectrometry (LC-MS/MS) with quadrupole-time-of-flight (Q-ToF) instruments can be performed in the scan mode on trypsinderived peptides with sufficient analytic accuracy to quantify synthesis rates of hundreds of proteins concurrently after relatively low-level in vivo ${ }^{2} \mathrm{H}_{2} \mathrm{O}$ labeling $(25,29-31)$.

Here, to address the third problem noted above, we report the development and validation of blood test approach for measuring the integrated rate of muscle protein synthesis over days to weeks. Our goal was to develop a translatable mass spectrometric technique for monitoring skeletal muscle protein synthesis rates noninvasively, from a single blood sample that reflects the dynamics of the skeletal muscle proteome under basal and anabolic conditions. This minimally invasive method can be seen as a virtual biopsy for monitoring skeletal muscle protein synthesis rates. We asked whether the labeling kinetics of proteins in plasma that were synthesized in skeletal muscle, such as creatine kinase M-type (CK-M) and carbonic anhydrase 3 (CA-3) the dynamics of the skeletal muscle proteome, using ${ }^{2} \mathrm{H}_{2} \mathrm{O}$ labeling combined with LC-MS/MS analysis (25, 29-33). In addition, the synthesis and turnover rates of multiple proteins across the proteome (i.e., proteome dynamics) had not previously been characterized in human skeletal muscle. We report that synthesis rates of proteins across the muscle proteome generally change in a coordinate manner in response to a sprint interval exercise training regimen in humans and to anabolic or catabolic interventions in rodents. Moreover, we report that labeling kinetics of plasma CK-M and CA-3 revealed these coordinate changes while detecting interindividual differences in muscle protein dynamics.

In combination with a technique for measuring skeletal muscle mass from a spot urine sample that we and our colleagues recently described (34-36), these translatable techniques have potential use as biomarkers in pharmacologic research and clinical management of disorders of skeletal muscle.

\section{Results}

\section{Preclinical studies}

Proteome dynamics in rat skeletal muscle after denervation and clenbuterol treatment. Proteome-wide synthesis rates were measured in rat gastrocnemius muscle by LC-MS/MS after 4 days of ${ }^{2} \mathrm{H}_{2} \mathrm{O}$ administration to determine the broad effects of 2 interventions that are well established to alter skeletal muscle mass denervation and treatment with clenbuterol $(20,37)$. Sciatic nerve denervation produced a significant $30 \%$ decrease in weight of gastrocnemius muscle in vehicle-treated groups (contralateral: $623.5 \pm 27.2 \mathrm{mg}$, denervation: $432.5 \pm 37.6 \mathrm{mg}, P<0.001)$, whereas clenbuterol treatment significantly increased the weight of muscle in denervated limb by $15 \%$ (vehicle: $432.5 \pm 37.6 \mathrm{mg}$, clenbuterol: $498.5 \pm$ $15.8 \mathrm{mg}, P<0.05$ ). Values represent mean $\pm \mathrm{SD}$ of 4 rats per group and were significantly different by 2 -way ANOVA.

FSRs met analytic criteria and were determined for each group ( $n=4$ per group) of animals, and there were $117 \pm 23$ proteins from the denervated limb, $114 \pm 25$ proteins from the contralateral limb, $122 \pm 26$ proteins from the vehicle-treated muscle, and $109 \pm 20$ proteins from the clenbuterol-treated muscle; the values represent the mean \pm SD of number of protein FSRs measured in individual rat per group. For 75 of the proteins, synthesis rates were measurable in all 4 groups and therefore could be compared; these are represented as a heatmap (Figure 1A). Unsupervised hierarchical clustering analysis of protein FSRs spontaneously organized animals into 4 groups, which were identical to the 4 treatment groups. In addition, a principal component analysis (PCA) of the data revealed that muscle FSRs were sufficient to differentiate each of the groups from the others (Supplemental Figure 1; supplemental material available online with this article; doi:10.1172/JCI79639DS1). Of special interest to our virtual biopsy approach, FSR of the cytosolic enzyme CK-M were significantly decreased by denervation and increased by clenbuterol treatment in both the contralateral and denervated limbs (Figure 1B). In contrast, FSR of another cytosolic enzyme, CA-3, was increased by clenbuterol treatment in contralateral limb and also increased by denervation (Figure 1C). FSRs of contractile myofibril proteins were significantly lower in the denervated limb compared with the contralateral limb, and treatment with clenbuterol significantly increased the FSRs of most proteins in both the denervated and control limbs (Figure 1D). A similar pattern was apparent for glycolytic enzymes, for which denervation uniformly decreased FSRs while clenbuterol increased FSRs (Figure 1E). Mitochondrial protein FSRs were significantly increased by clenbuterol only in the denervated limb but not in the contralateral limb, and they were not significantly altered by either clenbuterol or denervation alone (Figure $1 F)$. Thus, the FSR signatures for the clenbuterol (anabolic) versus denervation (catabolic) groups were not entirely symmetric across all of these ontologies.

Virtual biopsy: FSR of CK-M and CA-3 in rat muscle and plasma after clenbuterol treatment. Next, we asked whether muscle protein FSRs could be measured in a minimally invasive manner in rats based on the FSR of a protein isolated from the bloodstream. CK-M is a cytosolic enzyme predominantly (>90\%, ref. 38$)$ present in skeletal muscle that can be detected in serum or plasma and is routinely released from damaged muscle, which has led to its use as a biomarker of rhabdomyolysis (39). CA-3 is virtually specific to skeletal muscle in humans and can also be detected in circulation $(40,41)$. In rats, CA-3 is an abundant cytosolic enzyme in both muscle and liver (42). We hypothesized that the FSRs of CK-M or CA-3, synthesized in skeletal muscle and isolated from plasma after in vivo labeling, might provide a virtual biopsy of the FSR of skeletal muscle proteins.

FSR of plasma and skeletal muscle CK-M and CA-3 were measured in rats at 3,7 , or 14 days of ${ }^{2} \mathrm{H}_{2} \mathrm{O}$ labeling during clenbuterol treatment. At all time points, the fractional synthesis (f) of CK-M was higher in the gastrocnemius and quadriceps muscle 
A

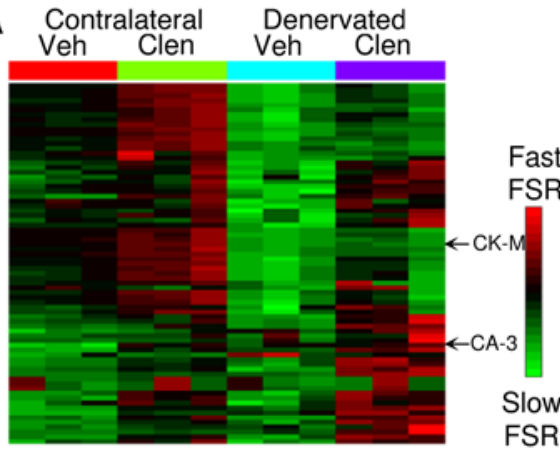

D

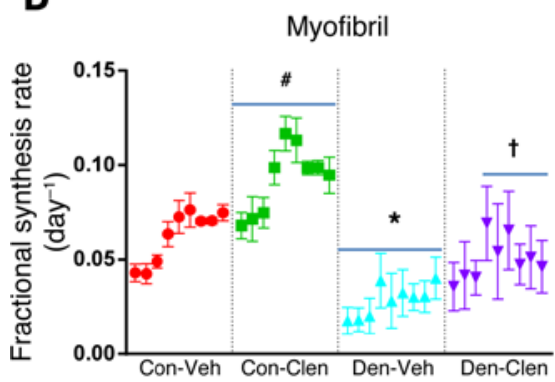

Actin, $\alpha$ skeletal muscle

Actin, cytoplasmic 2

Myosin regulatory light chain 2 , skeletal muscle isoform

Myosin light chain 1/3, skeletal muscle isoform

Tropomyosin, $\alpha-1$ chain

Tropomyosin, $\beta$ chain

Sarcoplasmic/endoplasmic reticulum

calcium ATPase 1

Sarcoplasmic/endoplasmic reticulum

calcium ATPase 2

Troponin I, skeletal muscle
B

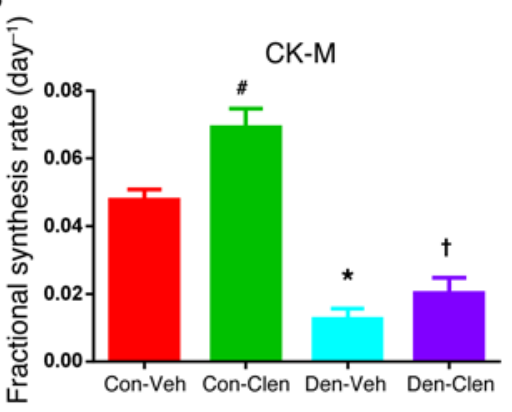

C

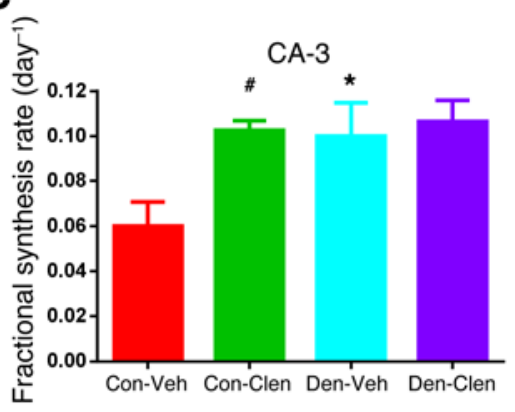

E

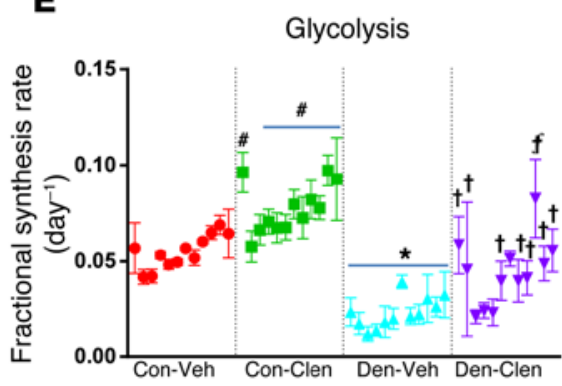

Glucose-6-phosphate isomerase

$\alpha$-enolase

$\gamma$-enolase

$\beta$-enolase

Phosphoglycerate mutase 2

Glyceraldehyde-3-phosphate

dehydrogenase

Phosphoglucomutase-1

Triosephosphate isomerase

Pyruvate kinase isozymes M1/M2

L-lactate dehydrogenase $B$ chain

Fructose-biphosphate aldolase $A$

L-lactate dehydrogenase $A$ chain

\section{$\mathbf{F}$}

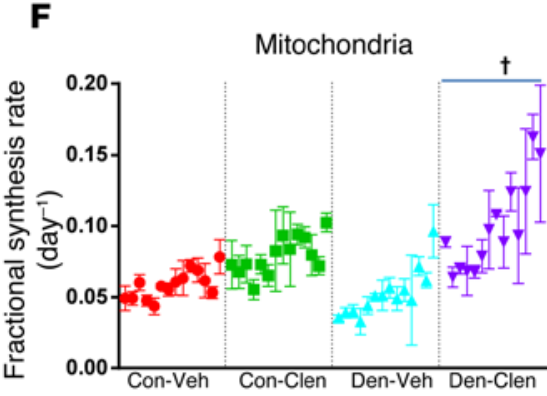

ATP synthase subunit e

Aconitate hydratase

ATP synthase subunit $\alpha$

ATP synthase subunit b

Protein DJ-1

Cytochrome c oxidase subunit $6 \mathrm{C}-2$

Phosphate carrier protein

Dihydrolipoyl dehydrogenase

Pyruvate dehydrogenase E1 component subunit $\beta$

2-oxoglutarate dehydrogenase

Electron transfer flavoprotein subunit $\alpha$

Superoxide dismutase [Mn]

Peroxiredoxin- 5

Thioredoxin-dependent peroxide reductase

Figure 1. Proteome dynamics in rat gastrocnemius muscle. (A) Heatmap of FSRs (\% day $\left.{ }^{-1}\right)$ of 75 proteins in rat muscle measured in $n=3$ rats per group after denervation and clenbuterol treatment, with each horizontal line representing z-scaled FSR of an individual protein. (B) Effect of denervation surgery and clenbuterol treatment on FSR of CK-M, mean \pm SD, $n=3-4$ /group. ${ }^{*} P<0.05$ 'Den-Veh' vs. 'Con-Veh', ${ }^{*} P<0.05$ 'Con-Clen' vs. 'Con $/$ Veh', ${ }^{\dagger} P<0.05$ 'Den-Clen' vs. 'Den-Veh', 2-way ANOVA. (C) Effect of denervation surgery and clenbuterol treatment on FSR of CA-3, mean \pm SD, $n=3-4 /$ group. ${ }^{*} P<0.05$ 'Den-Veh' vs. 'Con-Veh', " $P<0.05$ 'Con-Clen' vs. 'Con-Veh', 2-way ANOVA. (D-F) Effect of denervation surgery and clenbuterol treatment on the FSRs of myofibril, glycolytic, and mitochondrial proteins. Names are listed in the order they appear on the scatter plots for each class of proteins. Data represent mean \pm SD, $n=3-4$ /group, * corrected $P<0.05$ 'Den-Veh' vs. 'Con-Veh', \# corrected $P<0.05$ 'Con-Clen' vs. 'Con/Veh', ${ }^{\dagger}$ corrected $P<0.05$ 'Den-Clen' vs 'DenVeh', 2-way ANOVA with Holm-Sidak correction. Con, control; Veh, vehicle; Clen, clenbuterol; Den, denervation.

of clenbuterol-treated rats than in vehicle-treated animals (Figure 2, A and B). There was a highly significant correlation between $\mathrm{f}$ of muscle tissue CK-M and other muscle proteins measured in the tryptic digest of skeletal muscle samples (Supplemental Tables 1 and 2). The f of CK-M immunoprecipitated from plasma was also significantly increased after clenbuterol treatment (Figure 2C). Most importantly, there was a highly significant correlation $(r=\sim 0.98)$ between the f of CK-M isolated from plasma and the $\mathrm{f}$ of CK-M measured in gastrocnemius (Figure 2D) and quadriceps (Figure 2E) muscle tissue from the same animals, consistent with the principle that $>90 \%$ of plasma CK-M is derived from skeletal muscle (38). Plasma CK-M f also correlated highly significantly with the fof other proteins measured in the muscle samples (Supplemental Tables 1 and 2). Similar findings were observed for CA-3, with clenbuterol treatment producing a significant increase in $\mathrm{f}$ of CA-3 in both muscle and plasma compartments (Figure 3, A-C). Muscle tissue CA-3 values of $\mathrm{f}$ correlated highly significantly with $\mathrm{f}$ of other skeletal muscle proteins (Supplemental Tables 1 and 2) and with fof plasma CA-3 (Figure 3, D and E). The observations that both CK-M and CA-3 synthesis rates correlated well with synthesis rates of most proteins in muscle under anabolic conditions is consistent with the view that skeletal muscle tends to accrue structural and cytosolic proteins as an ensemble (43) and suggests that plasma CK-M and CA-3 synthesis rates should reflect the general synthesis rate of muscle structural and cytosolic proteins in the rat. 
A

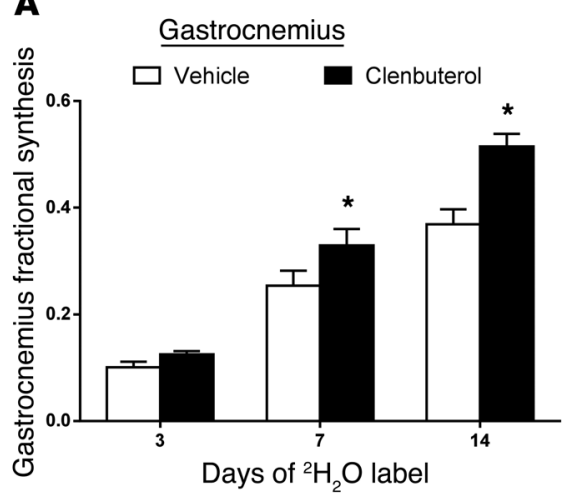

D

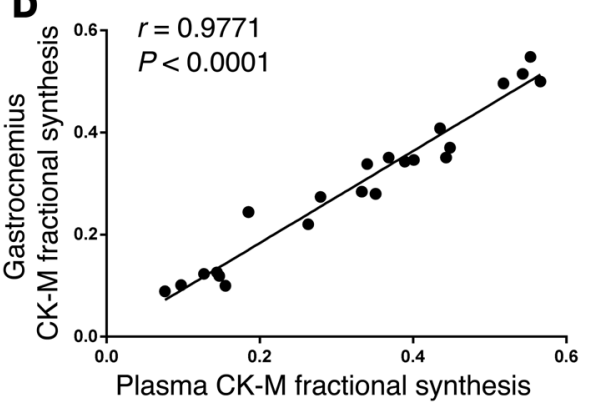

B

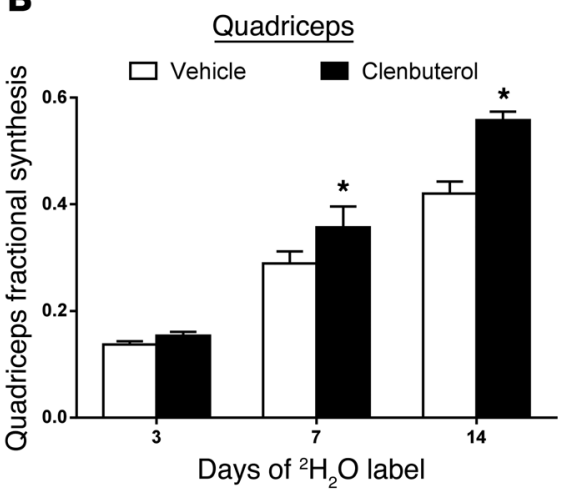

E
C

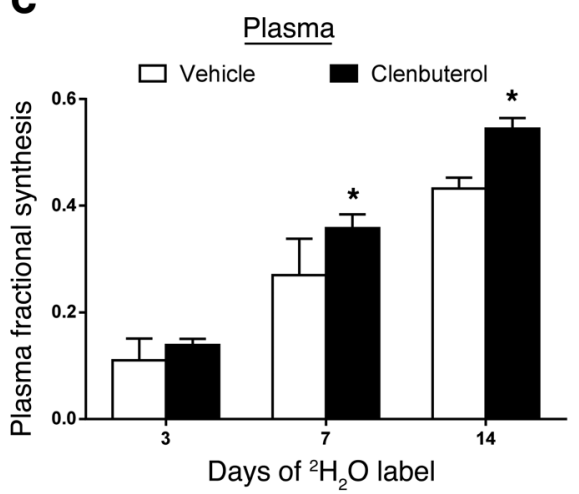

Figure 2. Effects of clenbuterol treatment on $\mathbf{f}$ of $\mathbf{C K}-\mathbf{M}$ in rat muscles and plasma. (A) CK-M $\mathrm{f}$ (\%) in gastrocnemius muscle of rats treated with clenbuterol for 3,7 , or 14 days (mean $\pm \mathrm{SD}, n=4$ /group, ${ }^{*} P<0.05$ clenbuterol vs. vehicle, 2 -way ANOVA). (B) CK-M f in quadriceps muscle of rats treated with clenbuterol for 3,7 , or 14 days (mean $\pm \mathrm{SD}, n=4$ /group, ${ }^{*} P<0.05$ clenbuterol vs. vehicle, 2 -way ANOVA). (C) CK-M $\mathrm{f}$ in plasma of rats treated with clenbuterol for 3, 7, or 14 days (mean \pm SD, $n=4$ /group, ${ }^{*} P<0.05$ clenbuterol vs. vehicle, 2 -way ANOVA). (D) Significant correlation (Pearson correlation $r>0.95, P<0.001$ ) between fractional syntheses of plasma CK-M compared with that of gastrocnemius CK-M. (E) Significant correlation (Pearson correlation $r>0.95, P<0.001$ ) between fractional syntheses of plasma CK-M compared with that of quadriceps CK-M.

\section{Human studies}

Proteome dynamics in human skeletal muscle: effects of sprint exercise. Proteome-wide synthesis rates were measured in quadricep muscle biopsies from 11 healthy human subjects, 6 of whom had gone through a sprint-interval exercise program as described elsewhere (25), while the other 5 subjects were sedentary controls. Subjects drank $50 \mathrm{ml}$ of ${ }^{2} \mathrm{H}_{2} \mathrm{O}$ (70\% enriched) twice daily for 3 weeks after an initial 1-week priming protocol of $50 \mathrm{ml} 3$ times a day. FSRs were measured for 515 proteins, of which FSRs for 273 proteins were measurable in $\geq 2$ subjects per group and could therefore be compared. FSRs ranged from a minimum of $2.2 \%$ per week to a maximum of $75 \%$ per week (Supplemental Table 3A). Sprint exercise produced a substantial increase in FSRs of most proteins analyzed. The FSRs of 139 individual proteins that were measured at least in $60 \%$ of the subjects in each group are shown in a heatmap (Figure 4A). Interindividual differences in FSR are apparent and were consistent across most protein through each subject's sampled proteome, reflecting a characteristic, individualized response to exercise. Pearson cross correlation coefficient analyses revealed that FSRs in muscle were more similar across proteins measured in sedentary subjects (average $r=0.883$ ) compared with subjects that participated in the sprint exercise program (average $r=0.722$ ).

The mean FSRs of proteins in sedentary and sprint groups were further compared at the gene ontological level. Using a data analysis program to group proteins based on their ontology terms accessed programmatically from the Database for Annotation, Visualization and Integrated Discovery (DAVID) v6.7 $(44,45)$, a paired $t$ test of protein FSRs by gene ontology was performed. A total of 8 nonredundant biological processes were enriched with significant differences $(P<0.05$ in paired $t$ tests for proteins, with Benjamini-Hochberg multiple test corrections) in mean protein FSR when comparing kinetics of the muscle proteome from subjects who were sedentary verses those who underwent sprint exercise. Proteins assigned to several gene ontologies were significantly affected following sprint exercise, including the 29 proteins classified as being involved in glucose metabolic processes, the 17 proteins involved in regulation of apoptosis, the 16 proteins involved in striated muscle contraction, and the 12 proteins involved in cellular respiration (Figure 4B); individual protein data are shown in Supplemental Table 3B. We also performed a between-group unpaired $t$ test on 67 proteins that were measured in all subjects. Following Benjamini-Hochberg multiple test corrections, there were 20 proteins that were significantly $(\mathrm{FDR}<0.2)$ different between the groups (Figure $4 \mathrm{C}$ ), reflecting the variability of protein metabolic response to exercise. The molecular kinetic findings of higher FSR changes in glycolytic enzymes and contractile proteins, compared with smaller changes in mitochondrial proteins, may provide insight regarding the functional consequences of this widely used sprint exercise program.

Virtual biopsy: CK-M and CA-3 FSRs in plasma compared with muscle in humans. Our primary goal in these studies was to develop 
A

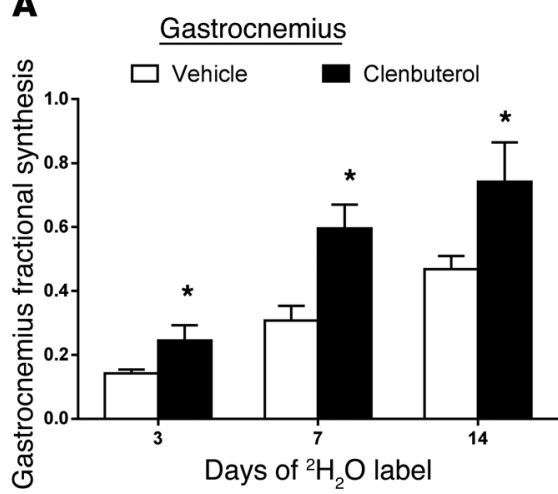

D

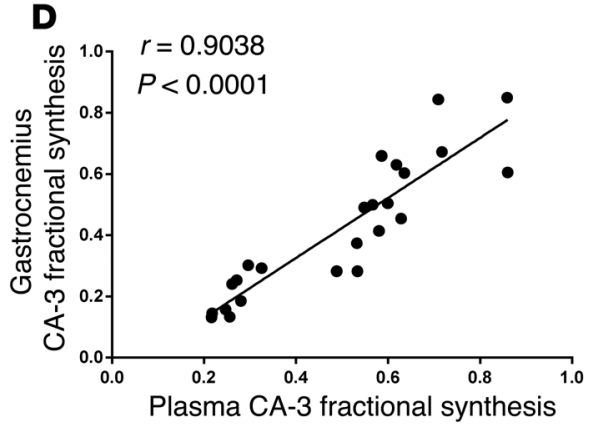

B

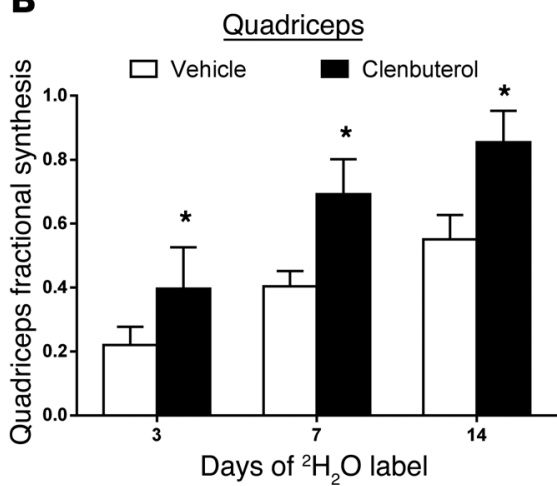

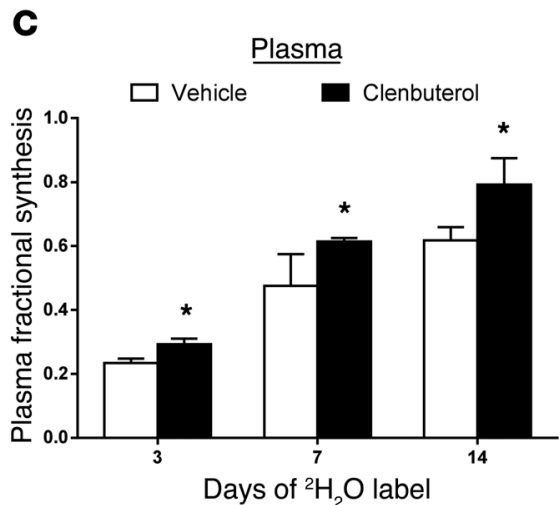

E

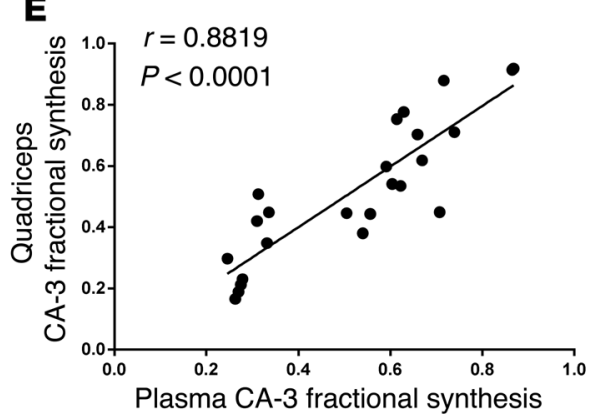

Figure 3. Effects of clenbuterol treatment on $f$ of CA-3 in rat muscles and plasma. (A) $C A-3 f(\%)$ in gastrocnemius muscle of rats treated with clenbuterol for 3,7 , or 14 days (mean $\pm S D, n=4$ /group, ${ }^{*} P<0.05$ clenbuterol vs. vehicle, 2 -way ANOVA). (B) CA-3 f in quadriceps muscle of rats treated with clenbuterol for 3,7 , or 14 days (mean $\pm S D, n=4$ /group, ${ }^{*} P<0.05$ clenbuterol vs. vehicle, 2-way ANOVA). (C) CA-3 f in plasma of rats treated with clenbuterol for 3 , 7, or 14 days (mean $\pm S D, n=4$ /group, ${ }^{*} P<0.05$ clenbuterol vs. vehicle, 2 -way ANOVA). (D) Significant correlation (Pearson correlation $r>0.9, P<0.001$ ) between fractional syntheses of plasma CA-3 compared with that of gastrocnemius CA-3. (E) Significant correlation (Pearson correlation $r>0.88, P<0.001$ ) between fractional syntheses of plasma CA-3 compared with that of quadriceps CA-3.

a method in humans for measuring skeletal muscle protein dynamics without the need for a muscle biopsy, i.e., from a measurement on a body fluid. Our finding in rats - that FSR of plasma CK-M and CA-3 reflect the FSRs of muscle CK-M, CA-3, and general glycolytic enzymes and cytoskeletal proteins, especially under anabolic conditions - was tested in human subjects. For these within-subject comparisons, we included 6 additional subjects (for a total of 17 subjects) from the same study who had received a dietary supplement (with or without sprint exercise), since we were interested in the relationship between plasma and muscle biopsy measurements in the same individuals and it is unlikely that the dietary supplement or its interactions with exercise would alter this relationship.

To establish the time course of a muscle-derived protein in plasma, we first measured the FSR of CK-M isolated from human plasma samples in 3 male subjects who ingested ${ }^{2} \mathrm{H}_{2} \mathrm{O}$ daily for 1-7 weeks. CK-M was immunoprecipitated from plasma using an anti-CK-M specific antibody, followed by gel fractionation, in-gel trypsin digestion, and LC-MS/MS analysis. The f of CK-M measured at each time point was fit to a monoexponential rise to plateau equation $\left(\mathrm{f}=1-\mathrm{e}^{-\mathrm{kt}}\right)$. The rate constant, $\mathrm{k}$, (synonymous with FSR) was $1.0 \% \pm 0.2 \%$ per day (Figure $5 \mathrm{~A}$ ). The curve fits were consistent with a plateau value close to $100 \%$, and any single time point collected between 1-7 weeks was sufficient to reveal the FSR value calculated by the curve fit (mean FSR from individual time points $=1.0 \% \pm 0.1 \%$ per day).
In order to determine whether plasma CK-M or CA-3 FSRs can be used as virtual biopsies of muscle CK-M, CA-3, and general protein synthesis rates in humans, FSR of CK-M and CA-3 were compared in plasma and a muscle biopsy specimen (vastus lateralis) for the 17 healthy subjects. All subjects drank ${ }^{2} \mathrm{H}_{2} \mathrm{O}$ for 4 weeks. CK-M FSR in human muscle ranged from 3.9\%-11.7\% per week in young male and female sedentary and exercising subjects compared with FSR in plasma of the same subjects of $4.5 \%-12.3 \%$ per week. There was a highly significant correlation between the FSR values of CK-M isolated from muscle and plasma $(r>0.89$, Figure $5 \mathrm{~B}$ ), similar to the observation in preclinical studies. Similarly, FSR of CA-3 in human muscle ranged from $2.8 \%-9.1 \%$ per week, compared with FSR in plasma of $4.4 \%-9.6 \%$ per week, with a very highly significant correlation ( $r>0.87$, Figure $5 \mathrm{C}$ ).

Correlation of CK-M and CA-3 FSRs with FSRs of human muscle proteins. We also determined the correlations between FSRs of CK-M and CA-3 in the muscle or plasma to the FSRs of other proteins in muscle. First, FSR of both muscle CK-M and CA-3 in the 17 subjects correlated closely and highly significantly $(r=0.84-0.94, P<0.0001)$ with that of the major myofibril proteins, such as actin, myosin, troponin, and tropomyosin in muscle tissue samples (Figures 6, A and B), as well as with other myofibril, 68 cytosolic, and 22 mitochondrial proteins (Supplemental Table 4). Moreover, FSRs of plasma CK-M and CA-3 also correlated significantly with the FSRs of major myofibrillar, cytosolic, and mitochondrial proteins in skeletal muscle (Figure 
A

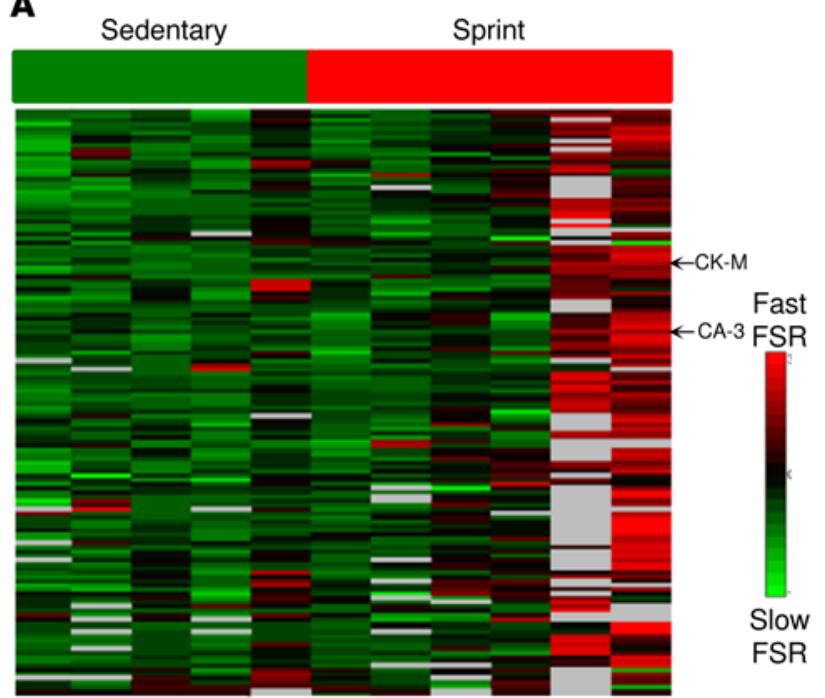

B

Glucose metabolic process

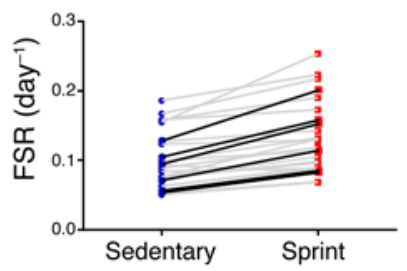

Striated muscle contraction

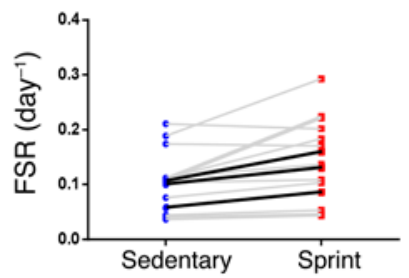

Regulation of apoptosis

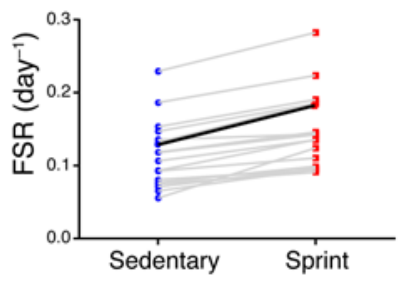

Cellular respiration

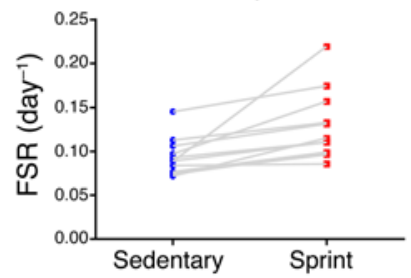

C

14-3-3 protein $\gamma$

Filamin-C

2-oxoglutarate dehydrogenase, mitochondrial Heat shock $70 \mathrm{kDa}$ protein $1 \mathrm{~A} / 1 \mathrm{~B}$ -

Fructose-bisphosphate aldolase A -

Myosin light chain 1/3, skeletal muscle isoform -

Fumarate hydratase, mitochondrial -

Pyruvate kinase isozymes M1/M2 -

ATP synthase subunit $\alpha$, mitochondrial -

Creatine kinase S-type, mitochondrial -

ATP synthase subunit $\beta$, mitochondrial L-lactate dehydrogenase $A$ chain -

Malate dehydrogenase, cytoplasmic -

Aspartate aminotransferase, cytoplasmic -

Glyceraldehyde-3-phosphate dehydrogenase Phosphoglycerate mutase 1 Glucose-6-phosphate isomerase Phosphoglycerate kinase 1 Phosphoglycerate mutase 2 Triosephosphate isomerase

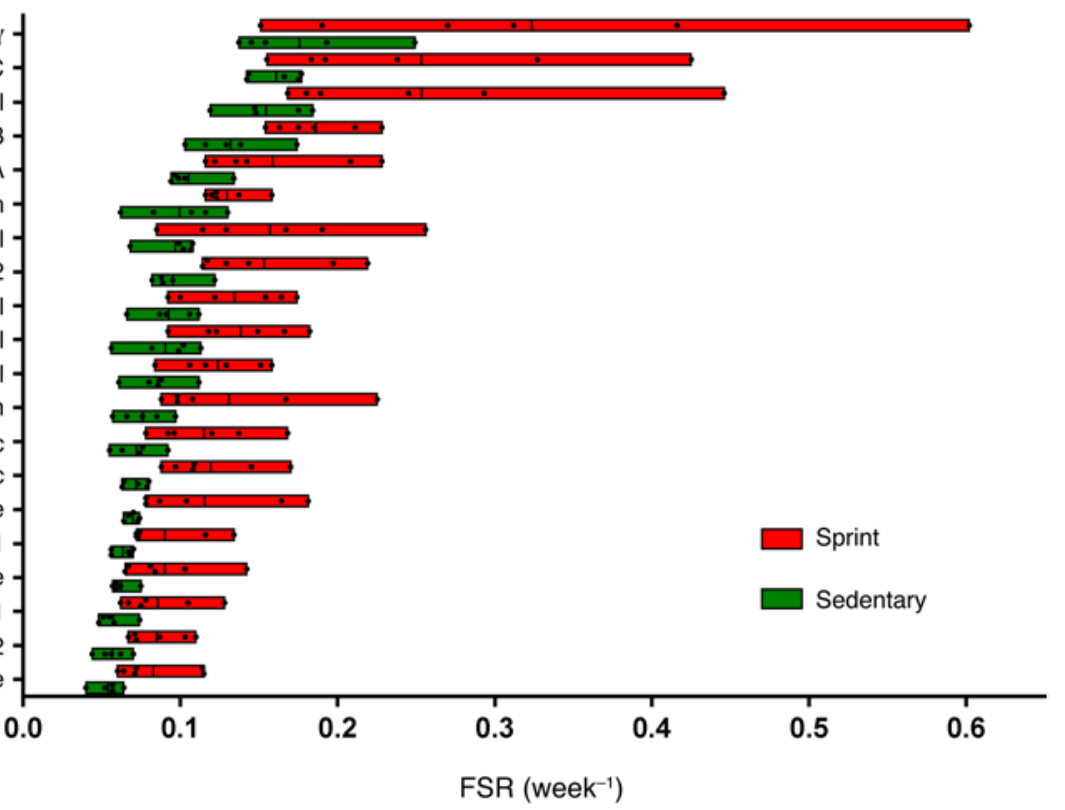

Figure 4. Human skeletal muscle proteome dynamics in sedentary and SIT subjects. (A) Heatmap of FSRs (\% week ${ }^{-1}$ ) of 139 proteins measured in muscle of 5 sedentary subjects and 6 subjects who underwent SIT. Each horizontal line represents an individual protein. (B) Mean FSR of proteins in DAVID gene ontology term, biological processes level 5 , that were significantly different as a group $(P<0.05$ in paired $t$ tests for proteins with Benjamini-Hochberg multiple test corrections) in sedentary $(n=3-5)$ or sprint $(n=4-6)$ subjects. Black lines reflect proteins whose FSRs were significantly different (uncorrected $P<0.05$, unpaired $t$ tests between subjects in the 2 groups) within the ontologies. For glucose metabolic process: phosphoglycerate mutase 2 ; fructose1,6-bisphosphatase isozyme 2; pyruvate kinase isozymes M1/M2; malate dehydrogenase cytoplasmic; triosephosphate isomerase; fructose-bisphosphate aldolase A; and phosphoglycerate kinase 1. For striated muscle contraction: Phosphoglycerate mutase 2, Fructose-bisphosphate aldolase A, and myosin light chain 1/3 skeletal muscle isoform. For regulation of apoptosis: heat shock $70 \mathrm{kDa}$ protein 1A/1B. (C) FSRs of 20 proteins that were significantly different between sedentary $(n=5)$ and sprint $(n=6)$ subjects (unpaired $t$ tests between the 2 groups with Benjamini-Hochberg multiple test corrections). For each protein, the bar represents mean, minimum, and maximum FSR; symbols within bars represent individual FSR values.

7, A and B, and Supplemental Table 4). Since 2 of the subjects in the sprint group had high FSRs, correlations were also performed excluding these subjects, and these remained significant $(r=\sim 0.6-0.8, P<0.05)$ for most proteins. These results indicate that in humans, as observed in rats, plasma CK-M and CA-3 synthesis rates reflect global muscle protein synthesis rates and detect the coordinated effects of an anabolic intervention on muscle protein dynamics.

\section{Discussion}

There were 2 primary goals of this work. The first was to develop a minimally invasive method for measuring integrated rates of skeletal muscle protein synthesis over a period of days to weeks based on a single blood measurement - i.e., without the need for a physical sample of the tissue. Muscle biopsies are not likely to be widely used in the clinical setting, so a blood test of muscle protein synthesis rates that could be monitored over time would be attractive 

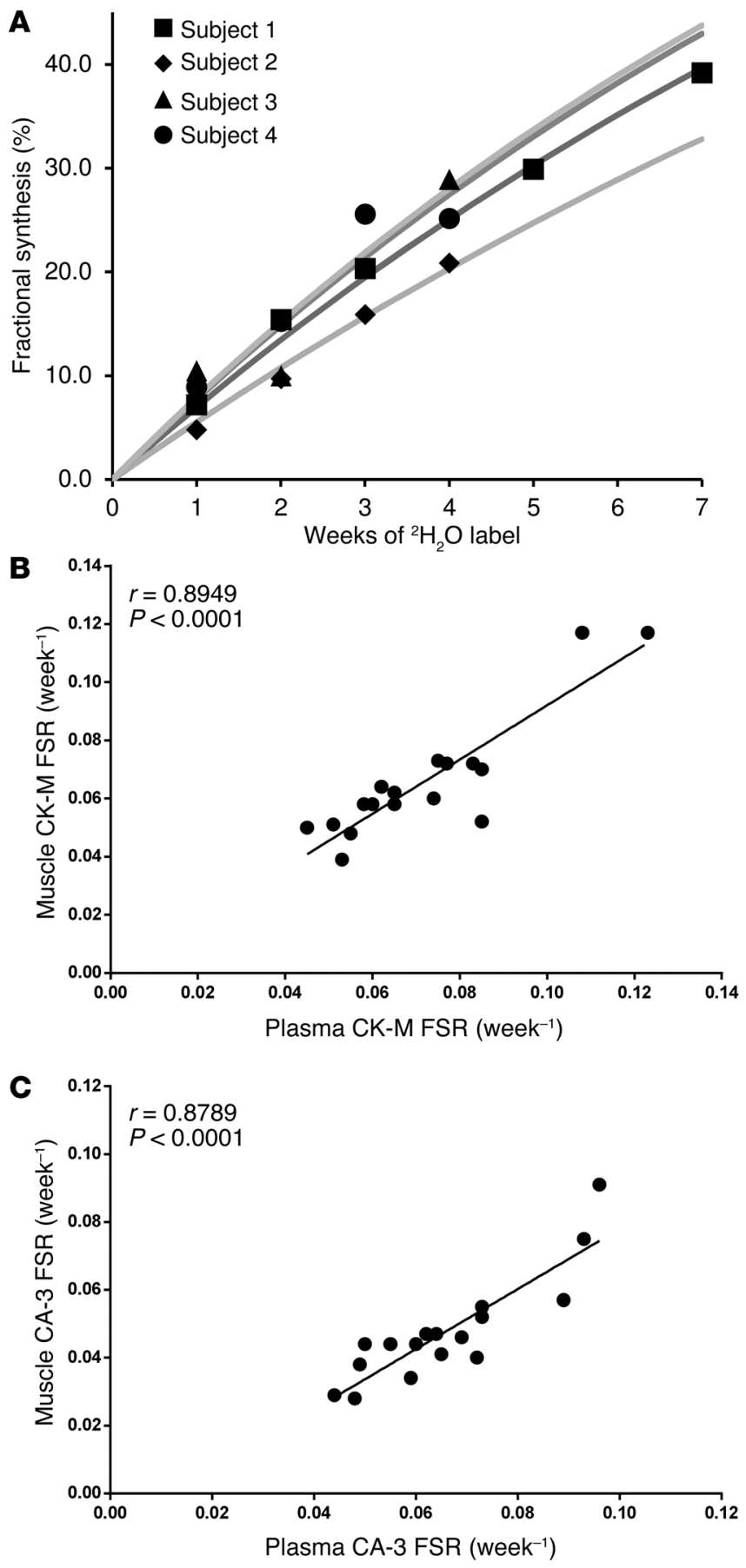

for drug development, as well as clinical management of disorders of muscle, including sarcopenia and cachexia. Because changes in muscle protein synthesis rates have been shown to occur rapidly in response to anabolic therapies and to predict subsequent changes in muscle mass, strength, and performance $(5,8-10)$, the availability of a blood test for skeletal muscle protein synthesis rates would greatly simplify translational drug development and monitoring of treatment efficacy in this growing, unmet medical need.

We describe here an approach that we call a virtual biopsy, based on a simple concept: if proteins can be identified in a body fluid that derive essentially exclusively from a single tissue, and if the rate-determining or slow kinetic step for such proteins is
Figure 5. CK-M and CA-3 synthesis in human plasma and skeletal muscle. (A) The $\mathrm{f}(\%)$ of CK-M in 4 subjects after continuous ${ }^{2} \mathrm{H}_{2} \mathrm{O}$ labeling for several weeks. (B) Significant correlation (Pearson correlation $r=0.89, P<0.0001$, $n=17$ ) between FSR (\% week ${ }^{-1}$ ) of CK-M measured in the plasma and in the skeletal muscle of the same subject. (C) Significant correlation (Pearson correlation $r=0.88, P<0.0001, n=17$ ) between FSR of CA-3 measured in the plasma and in the skeletal muscle of the same subject.

in the tissue (i.e., the half-life in the body fluid is shorter than in the tissue of origin), then the measured FSR of the protein isolated from the body fluid will reflect the FSR of the protein in the tissue of origin.

Here, we chose CK-M and CA-3 as candidate proteins to represent skeletal muscle protein turnover. CK-M and CA-3 were attractive candidates in principle because these circulating proteins are known to be $>90 \%$ derived from skeletal muscle in humans (38, $40,41)$, and their clearance from plasma is relatively fast in healthy subjects (46) while their half-lives in muscle are slow ( 2 months, as shown in Figure 4). These assumptions needed to be validated experimentally in rodents and humans. We show that FSR of muscle CK-M and plasma CK-M are essentially identical, and this is also true for FSR of muscle CA-3 and plasma CA-3, meeting the central principle of a virtual biopsy. Perhaps more importantly, FSRs of plasma and muscle CK-M and CA-3 correlated highly with FSRs of a large number of muscle proteins across multiple gene ontologies. The latter finding is consistent with the concept that muscle cytosolic and structural proteins tend to be synthesized as an ensemble when muscle mass increases or diminishes; i.e., the proportional content of muscle cells does not change when mass changes under anabolic and catabolic conditions (43). Repeated measurements of CK-M synthesis on blood samples at different time points gave reproducible and consistent results in subjects at steady-state for muscle mass, suggesting that monitoring of muscle FSR over time should be feasible to track the early effect of interventions directed at increasing muscle mass. The coordinated assembly of muscle proteins (43) and the confluence of muscle protein synthesis rates further supports the idea of using a representative and accessible protein, such as CK-M or CA-3 in plasma, to represent general protein dynamics in muscle.

It should be noted that CK-M and CA-3 are only 2 possible candidates for muscle-derived proteins that can be isolated from plasma to measure tissue protein synthesis rates, and they are particularly useful to track effects of anabolic interventions. In as much as no single protein, whether in the cell or in the circulation, can serve as the sole read-out for changes in all classes of muscle protein turnover, these cytosolic proteins may not accurately reflect all the protein kinetic changes in other compartments, such as mitochondria, especially when there are differential subcellular effects of interventions. There may be several other blood proteins that are similar or superior for this virtual biopsy purpose, making it feasible to identify a panel of blood proteins for monitoring different aspects of muscle protein dynamics. The finding that 2 different proteins in plasma that are believed to derive primarily from skeletal muscle in fact showed very similar FSRs in plasma and muscle strongly supports the general principle of virtual biopsy and suggests that other proteins will likely be identified. Interestingly, it may be possible to measure FSRs of muscle-specific mitochondrial proteins in the 

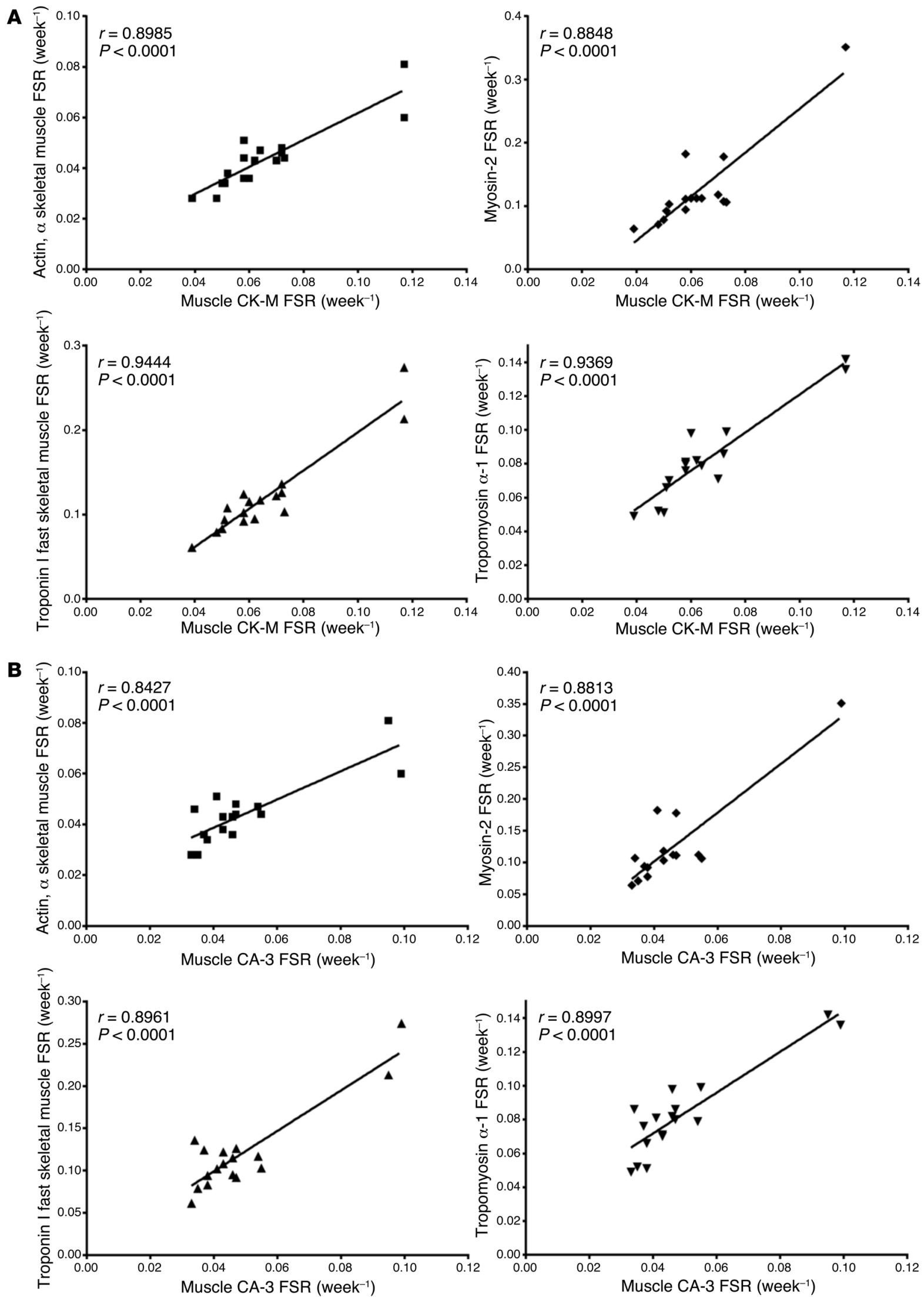

Figure 6. Muscle CK-M and CA-3 synthesis correlate with skeletal muscle protein synthesis. (A) Significant correlation (Pearson correlation $r>0.8$, $P<0.0001, n=16-17)$ between FSR (\% week $\left.{ }^{-1}\right)$ of CK-M and FSRs of myofibrillar proteins, both measured in skeletal muscle of the same subject. (B) Significant correlation (Pearson correlation $r>0.8, P<0.0001, n=16-17$ ) between FSR of CA-3 measured in plasma and FSRs of myofibrillar proteins measured in skeletal muscle of the same subject. 
circulation: 2 peptides from COX8B have been identified only in skeletal and cardiac muscle of mice (MitoCarta1.0 database, ref. 47). Although this protein has not been identified in humans, 2 peptides from COX6A2, which is present in humans, were also identified as muscle specific among the 14 mouse tissues studied (MitoCarta2.0 database, ref. 48). We have recently described a similar virtual biopsy approach for measuring hepatic fibrogenesis rates from blood samples by measuring FSRs of extracellular matrixrelated proteins (such as lumican or TGF $\beta$-induced protein) that escape from liver into the circulation (49). We believe that the underlying concept presented here may have broad applications, building on the great sensitivity of tandem mass spectrometry when combined with stable isotope labeling.

It is important to point out the differences between measuring concentrations versus synthesis rates of plasma proteins derived from muscle. Conceptually, the level of a protein in plasma has no fundamental relationship to its synthesis rate back in the tissue of origin but is a function of factors, such as release rate from the tissue and clearance rate from the blood. Because mass spectrometric analysis compares an internal ratio among different masses within a molecule, yield or recovery of the molecule do not, in principle, affect kinetic calculations and are therefore not subject to influence of tissue leakage or clearance from plasma. This feature provides a real advantage of kinetic measurements for blood-based assays.

Our second objective was to measure the synthetic rates of large numbers of proteins reproducibly across a variety of gene ontologies in muscle tissue from humans and experimental animals, in response to anabolic or catabolic interventions. This goal required an analytic approach that goes beyond small numbers of targeted proteins measured one at a time by LC-MS/MS (50-52). We have found that Q-ToF MS/MS instruments achieve the highest analytic accuracy and precision for quantifying mass isotopomer ratios in hundreds or thousands of peptides concurrently, which allows accurate and reproducible protein kinetic measurements from a single time point $(25,29-31)$. Using this approach, we have identified characteristic proteome dynamic signatures of several conditions, including calorie restriction in liver (31), experimental fibrosis in lung (29), proliferating neural stem cells in vitro (53), neuroinflammation in mouse brain and cerebrospinal fluid (54), and FSRs of human plasma proteins (30), but the detailed proteome dynamic response in human muscle to anabolic or catabolic conditions had not previously been measured.

We demonstrate here analytically robust kinetic measurements, as evidenced by the consistent changes within subjects and within functionally related classes of proteins in response to interventions. The labeling protocol is relatively nonburdensome, involving oral intake of ${ }^{2} \mathrm{H}_{2} \mathrm{O}$ in drinking water for 4-7 days in rodents and 2-3 daily doses for 1-4 weeks in humans. We report on the turnover rates of approximately 500 proteins in human skeletal muscle and approximately 100 proteins in rat skeletal muscle. The former number is larger in part because of differences in protein identification due to a larger Swiss-Prot human proteome database and also because we used a more sensitive model of the instrument (Q-ToF 6550) for the human analyses.

Technically, stringent analytic accuracy for isotope ratio measurements is required to quantify relatively low levels of deuterium incorporation during ${ }^{2} \mathrm{H}_{2} \mathrm{O}$ administration in humans and preclinical models $(25,29-31)$. Because the stringent analytic filtering criteria we have found are required for reliable kinetic measurements (30), the proteome-wide kinetic measurements described here using Q-ToF instruments quantify fewer proteins than can be identified with static proteomic measurements of protein content, especially when highly sensitive Orbitrap instruments are used for the latter (55). This is because a large fraction of identified peptides do not meet analytic criteria for quantitative accuracy of isotope ratio measurements. In addition, Orbitrap instruments to date have not been able to achieve sufficiently accurate isotope ratio measurements on peptides in the scan mode (56) to allow usable proteome-wide kinetic measurements at the low level deuterium enrichments that are achieved in human studies, which is why we have developed our current proteome dynamic approach using Q-ToF instruments. Having kinetic values for up to several hundred proteins in a tissue spans a wide variety of gene ontologies and functions, however (Figures 1 and 3 and refs. 29, 31) and allows broad conclusions about cellular response.

Earlier studies have documented a reduction in mixed muscle protein synthesis rates and content in skeletal muscle tissue (20, 57 ) or in mixed myofibril protein subfractions (37), after denervation, using radioactive amino acid tracers. More recent proteomic approaches have quantified changes in the abundances of specific proteins (58-60). Our quantitative profile of FSR changes of $>100$ specific proteins in response to denervation extend these findings and are consistent with gene expression changes in denervated muscle reported for structural proteins, enzymes involved in glycolysis, and mitochondrial ATP synthesis (61). Interestingly, the protein anabolic dynamic signature for clenbuterol treatment was not in all respects reciprocal to the signature for denervation. Clenbuterol produced a strong response of increased FSRs for cytosolic and structural proteins, as expected $(20,57)$, but its effects on mitochondrial proteins were limited to only the denervated limb, suggesting differential mechanisms and a stronger anabolic response under conditions of muscle atrophy. In addition, consistent with previous reports that denervation induces CA-3 in rat skeletal muscle $(62,63)$, we also observed that denervation increased CA-3 synthesis, whereas most cytosolic protein FSRs were reduced. Accordingly, in denervated rats, muscle or plasma CA-3 synthesis rates are not representative of reduced synthesis rates of most muscle cytosolic proteins.

In humans, the proteome dynamics revealed somewhat unexpected results for the skeletal muscle proteostatic response to sprint interval training (SIT), a program that involves repetitive 30 -second bouts of intensive exercise $(64,65)$. While several reports have documented increased glycolytic and oxidative enzyme activities in response to SIT (64-67), our results indicate that higher synthesis rates were observed for glycolytic enzymes and cytoskeletal proteins but that mitochondrial proteins in general did not exhibit higher FSRs in subjects on sprint compared with sedentary regimens. These protein dynamic findings provide molecular kinetic insight into the physiologic consequences of this exercise training regimen, suggesting a primary functional impact on the synthesis of proteins related to muscle anaerobic power and contractile strength. Proteins involved in regulation of apoptosis and oxidative stress, including heat- 
A
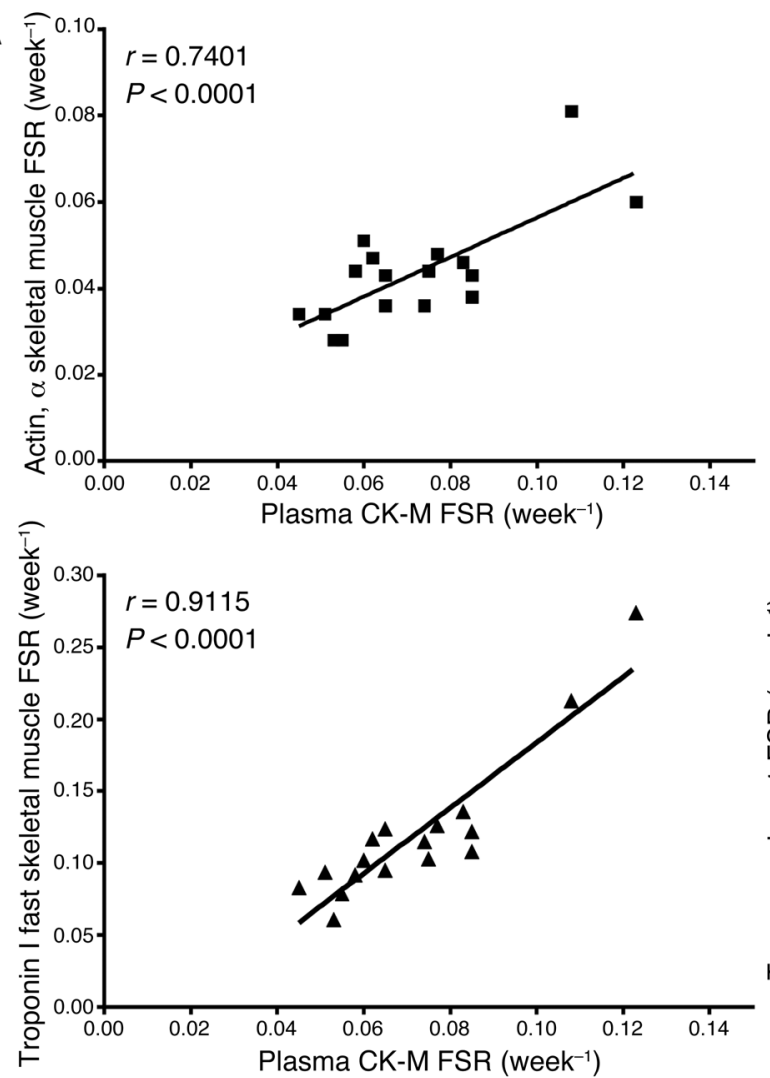
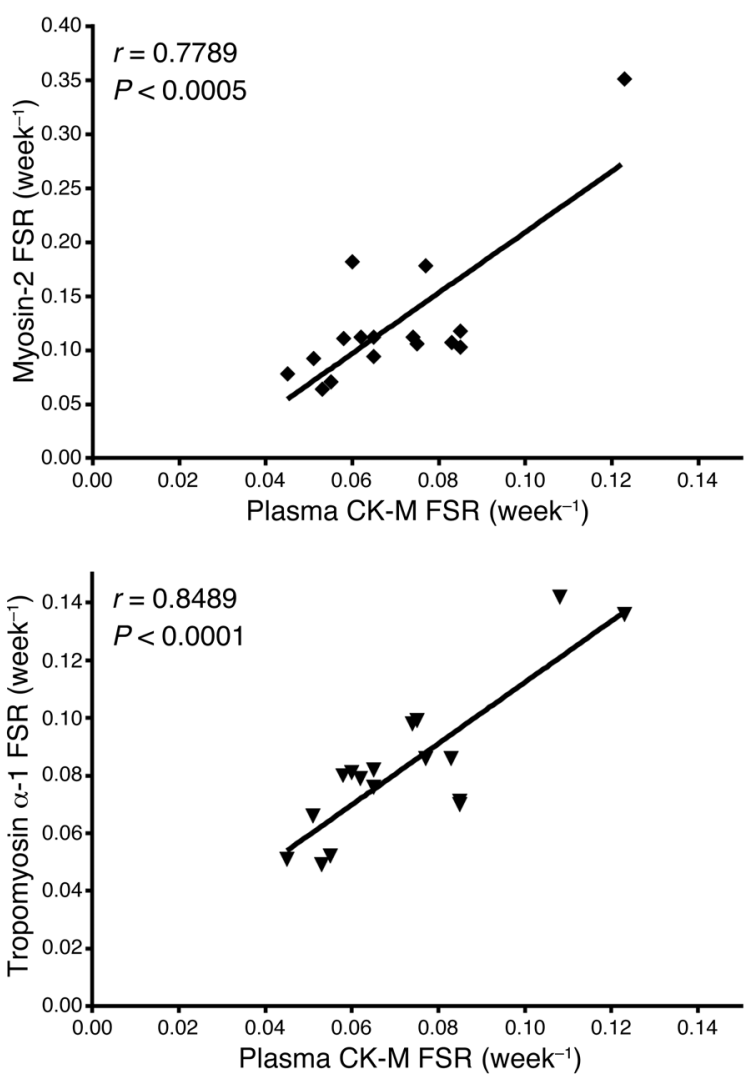

B

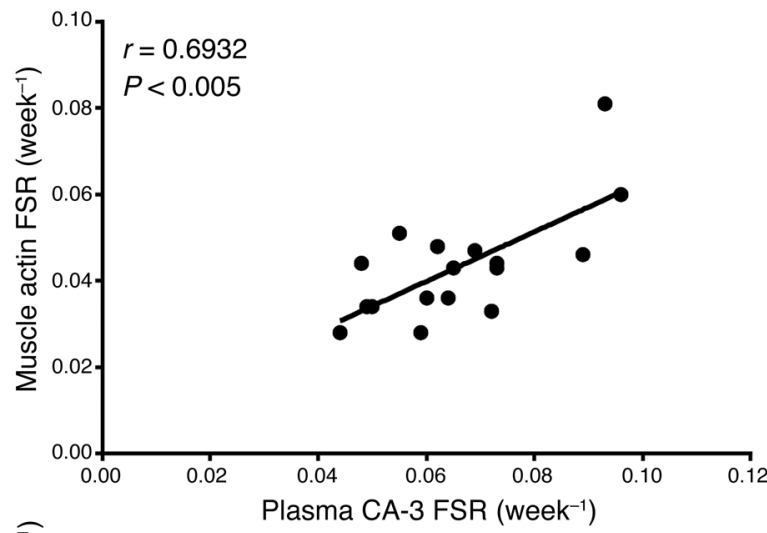

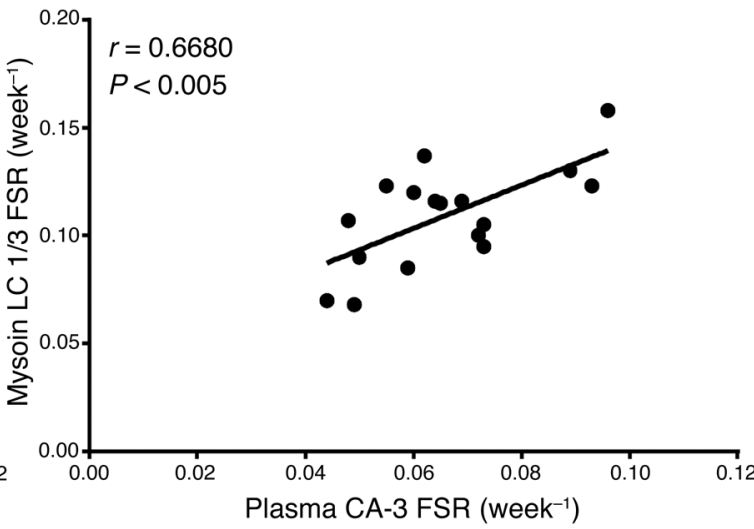

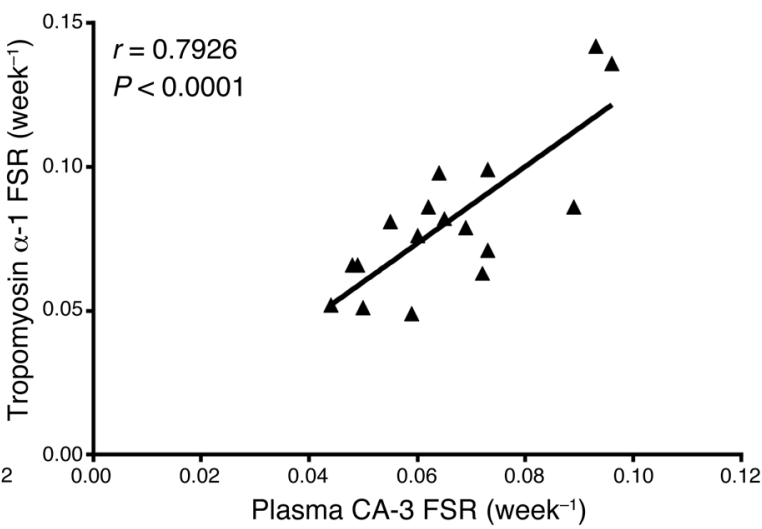

Figure 7. Plasma CK-M and CA-3 synthesis rates provide a virtual biopsy of skeletal muscle protein synthesis. (A) Significant correlation (Pearson correlation $r>0.7, P<0.0005, n=16-17)$ between FSR (\% week $\left.{ }^{-1}\right)$ of CK-M measured in the plasma and FSRs of myofibrillar proteins measured in skeletal muscle of the same subject. (B) Significant correlation (Pearson correlation $r>0.7, P<0.0005, n=16-17$ ) between FSR of CA-3 measured in plasma and FSRs of myofibrillar proteins measured in skeletal muscle of the same subject. 
shock proteins and antioxidant enzymes, also showed increased synthesis after SIT. These kinetic findings are in agreement with previous studies $(68,69)$ showing exercise-induced upregulation expression for these proteins.

Human muscle global proteome synthesis rates revealed not only group effects but also interindividual differences in response to sprint exercise. The observation of interindividual variability for response to exercise is well known at the macroscopic level in humans (70). Some sprint-exercise subjects exhibited markedly elevated FSR values across the proteome, compared with sedentary subjects, whereas other subjects' FSRs were barely different from sedentary subjects, even though all individuals were exposed to a similar supervised exercise program. Importantly, measurement of FSR in plasma CK-M accurately revealed individualized muscle protein synthetic response to exercise in human subjects or to anabolic interventions in rodents.

Limitations of this study include the relatively small number of human subjects studied, the cross-sectional rather than longitudinal comparison of exercise effects, and the broad proteome measurements comprising only hundreds of the thousands of proteins present in muscle tissues (71). The human study sample size was sufficient, however, to observe significant differences and to document considerable intersubject variability for response to exercise. The lower number of proteins with kinetic measurements was due to the stringent criteria imposed for data inclusion and the need for Q-ToFs rather than FT-ICR instruments to quantify isotope ratios in the scan mode. Longitudinal intervention studies may show even greater effects of exercise interventions by correcting for baseline variability among individuals. Finally, measurement of FSRs of 100-500 proteins across multiple gene ontologies revealed clear and consistent patterns, although it will be interesting to look more deeply into the proteome to identify selectivity of response among subjects of proteins.

Measurement of muscle protein synthesis rates may have important uses in translational drug development and clinical management. Changes in the FSR of muscle proteins have been reported within 5 days of starting anabolic treatments (8) and predict subsequent changes in muscle mass and strength in humans $(5,9)$ and rodents $(72)$, while the latter typically require 4-20 weeks to be observable. Indeed, a general principle of dynamic systems is that changes in synthesis or breakdown rates within a molecular pool typically precede and are more sensitive than changes in the pool size (73). Accordingly, measurements of protein kinetics may prove useful as rapid read-outs of target engagement and treatment efficacy in drug development or patient management, to identify early on whether an intervention is working. The availability of a blood test for monitoring muscle protein FSR potentially makes this an even more attractive approach. In addition, by combining the virtual biopsy plasma CK-M measurement of muscle protein synthesis with the recently described spot urine test of muscle mass (34), it may now be possible to gain insight into present status (mass) and future trajectory (synthesis rate) of muscle in settings of anabolic therapy or catabolic illness.

In summary, we have developed and validated a blood test for measuring the integrated rate of muscle protein synthesis over days or weeks. The test is based on label incorporation from ${ }^{2} \mathrm{H}_{2} \mathrm{O}$ into plasma CK-M or CA-3, proteins that are synthesized in skeletal muscle and escape into the bloodstream. The FSRs of plasma CK-M and CA-3 both correlate extremely well with FSR not only of muscle tissue CK-M and CA-3, but also with muscle proteins across many gene ontologies, measured by LC-MS/MS, in both humans and animal models. These findings strongly support the assumptions of the virtual biopsy model and provide a minimally invasive translational biomarker of skeletal muscle protein synthesis rates. The blood-based approach described here, alone or in combination with tissue-based proteome-wide measurements, have a number of possible uses in drug development and for advancing our understanding of nutritional and exercise effects on muscle biology, as well as potentially for medical diagnosis, prognosis, and monitoring.

\section{Methods}

Animals. Male Sprague-Dawley (SD) rats (250-300 g; Charles River Laboratories), were used for these studies. Animals were housed in a climate-controlled environment with a 12-hour light/dark cycle and were fed standard rodent chow and water ad libitum.

Surgery. Sciatic denervation surgery was used as a model for muscle atrophy and wasting as previously described (74). Animals were placed under isoflurane anesthesia, and buprenorphine was administered at the time of surgery. The sciatic nerve of one leg was exposed in the thigh region by blunt forcep reflection of the muscle. One hind limb was denervated with a hemostat by crushing the sciatic nerve immediately proximal to the division of the peroneal and tibial branches for 30 seconds. The skin was closed with wound clips. Denervation was known to be complete by observing postoperative disuse of the lower limb and loss of a withdrawal response to a pinch in the sciatic distribution area. The contralateral leg was sham operated, and its muscles were used as internal controls for the denervated tissues.

Drug treatment. Animals ( $n=4$ per group) started drug treatment by oral gavage 3 days after denervation surgery with either vehicle (water) or clenbuterol $\mathrm{HCl}(4 \mathrm{mg} / \mathrm{kg}$ ) dosed once daily for 4 days. In a separate time-course experiment, rats were gavaged daily with vehicle (water) or clenbuterol $\mathrm{HCl}(4 \mathrm{mg} / \mathrm{kg}$ ) for either 3, 7, or 14 days.

${ }^{2} \mathrm{H}_{2} \mathrm{O}$ labeling protocol in rats. Animals were labeled with ${ }^{2} \mathrm{H}_{2} \mathrm{O}$ concurrent with vehicle or clenbuterol treatment, which was 4 days in the denervation study and either 3, 7, or 14 days in the time-course study. Animals received a priming i.p. bolus of $35 \mathrm{ml} / \mathrm{kg} 0.9 \% \mathrm{NaCl}$ in $99.9 \%$ ${ }^{2} \mathrm{H}_{2} \mathrm{O}$ and were maintained on $8 \%{ }^{2} \mathrm{H}_{2} \mathrm{O}$ in drinking water until sacrifice. This regimen results in steady-state values of about $5 \%$ excess ${ }^{2} \mathrm{H}$ enrichment of body water.

Tissue harvesting. Animals were euthanized by $\mathrm{CO}_{2}$ asphyxiation. Blood was drawn by cardiac puncture and centrifuged at 2,000 $g$ for 10 minutes to obtain plasma, which was stored at $-20^{\circ} \mathrm{C}$ until processing. The gastrocnemius and quadriceps muscles were dissected out of each leg and stored at $-20^{\circ} \mathrm{C}$ until further processing.

${ }^{2} \mathrm{H}_{2} \mathrm{O}$ labeling protocol in humans. Deuterium labeling of newly synthesized proteins was achieved by oral consumption of ${ }^{2} \mathrm{H}_{2} \mathrm{O}$ (70\%; Cambridge Isotope Laboratories) for 4 weeks using protocols described previously $(24,25,30)$. A target of $1 \%-2 \%$ enrichment was achieved during a 1-week priming stage, when subjects consumed 50 $\mathrm{ml}$ of $70 \%{ }^{2} \mathrm{H}_{2} \mathrm{O}$ three times a day for a total of $150 \mathrm{ml} /$ day, which was maintained for 3 weeks with a dose of $50 \mathrm{ml}$ of $70 \%{ }^{2} \mathrm{H}_{2} \mathrm{O}$ two times a day for a total of $100 \mathrm{ml} /$ day. Subjects in the time-course study consumed $50 \mathrm{ml}$ of $70 \%{ }^{2} \mathrm{H}_{2} \mathrm{O}$ three times a day for a total of $150 \mathrm{ml} /$ day, 
which was maintained for a maximum of 7 weeks with a dose of $40 \mathrm{ml}$ of $70 \%{ }^{2} \mathrm{H}_{2} \mathrm{O}$ two times a day for a total of $80 \mathrm{ml} /$ day.

SIT. Participants completed 9 sessions of SIT over 3 weeks. Each session consisted of four to eight 30-second bouts of maximal efforts on a stationary cycle ergometer (Velotron, RacerMate Inc.) against a resistance equal to $7.5 \%$ of participant body mass separated by 4 minutes of active recovery; to facilitate recuperation, each session was separated by 1-2 days. The first and ninth sessions both consisted of four 30-second bouts to allow for comparisons to be made between these sessions.

Sample collection. Saliva swabs were collected periodically during oral ${ }^{2} \mathrm{H}_{2} \mathrm{O}$ consumption and stored at $-80^{\circ} \mathrm{C}$ until analysis. Venous blood was collected either during weekly blood draws or at the end of SIT training, and plasma was separated by centrifugation and stored at $-80^{\circ} \mathrm{C}$. While the subjects were under local anesthesia ( $1 \%$ lidocaine), muscle biopsy samples $(\sim 100-150 \mathrm{mg})$ of the vastus lateralis were removed using a 5-mm Bergström needle with manual suction and then immediately frozen in liquid nitrogen and stored at $-80^{\circ} \mathrm{C}$.

Body water enrichment analysis. Body water enrichment was determined from saliva swabs or plasma samples in humans and in rat plasma samples. Aliquots of plasma or saliva were diluted 1:200 and placed into the caps of inverted sealed screw-capped vials for overnight distillation at $80^{\circ} \mathrm{C}$. Body water ${ }^{2} \mathrm{H}_{2} \mathrm{O}$ enrichments were determined by direct measurement of deuterium mole percent excess (MPE) in water distilled from the blood plasma. MPE was measured against a ${ }^{2} \mathrm{H}_{2} \mathrm{O}$ standard curve using laser water isotope analyzer (LGR).

SDS-PAGE fractionation, Coomassie blue staining, and in-gel trypsin digestion of muscle proteins. Human or rat muscle tissues were prepared for LC/MS analysis using methods described previously (31). Briefly, 10-30 mg muscle samples were homogenized in M-PER reagent (Thermo Scientific) at $100 \mathrm{mg} / \mathrm{ml}$ with $1 \times$ protease inhibitor cocktail (Thermo Scientific). Protein $(250 \mu \mathrm{g})$ from prepared homogenates was uniformly reduced via incubation in $5 \mathrm{mM}$ Tris(2-carboxyethyl)phosphine (TCEP) and SDS-PAGE sample loading buffer for 5 minutes at $95^{\circ} \mathrm{C}$, alkylated via incubation in $15 \mathrm{mM}$ iodoacetamide for 1 hour at room temperature, and fractionated by SDS-PAGE (Invitrogen). The gel bands corresponding to 10 discrete molecular weight regions were excised from Coomassie blue-stained gels and digested overnight with trypsin (Proteomics Grade, Sigma-Aldrich) at $37^{\circ} \mathrm{C}$. The peptides were extracted from the gel, dried, and reconstituted in $3 \%$ acetonitrile/ $0.1 \%$ formic acid for LC/MS analysis.

Immunoprecipitation of CK-M and CA-3 from plasma, SDS-PAGE fractionation, and in-gel trypsin digestion. CK-M was immunoprecipitated from $500 \mu \mathrm{l}-2 \mathrm{ml}$ rat or human plasma using $20 \mu \mathrm{g}$ of goat anti-CK-M polyclonal antibody (CalBioreagents, P195) conjugated to $1 \mathrm{mg}$ epoxy Dynabeads (Invitrogen). Samples were incubated for 60 minutes at room temperature (RT), and the bound CK-M was eluted in sample buffer and prepared for SDS-PAGE fractionation and Coomassie blue staining as described above. The gel bands corresponding to $37-50 \mathrm{kD}$ were excised and subjected to overnight trypsin (Proteomics Grade, Sigma-Aldrich) digestion at $37^{\circ} \mathrm{C}$. The peptides were extracted from the gel, dried, and reconstituted in $3 \%$ acetonitrile/0.1\% formic acid for LC/MS analysis. CA-3 was immunoprecipitated from $2 \mathrm{ml}$ rat or human plasma using $20 \mu \mathrm{g}$ of goat anti-CA-3 polyclonal antibody (R\&D Systems, AF2185) conjugated to $1 \mathrm{mg}$ epoxy Dynabeads (Invitrogen). Samples were incubated for 60 minutes at RT, and the bound CA-3 was eluted in $30 \%$ acetonitrile, $0.5 \%$ formic acid ( $\mathrm{pH} \sim 2.5$ ), followed by in-solution trypsin digestion for LC/MS analysis.

LC/MS analysis. Trypsin-digested peptides were analyzed on a 6520 or 6550 Q-ToF mass spectrometer with 1260 Chip Cube nano ESI source (Agilent Technologies). Peptides were separated using a Polaris HR chip (Agilent Technologies, G4240-62030) consisting of enrichment and analytical columns packed with Polaris C18-A stationary phase. Mobile phases were $5 \% \mathrm{v} / \mathrm{v}$ acetonitrile and $0.1 \%$ formic acid in water and $95 \%$ acetonitrile and $0.1 \%$ formic acid in water. Peptides were eluted at $350 \mathrm{nl} /$ minute flow rate with an $18 \mathrm{~min}$ utes LC gradient (for IP samples) or a 27 minutes LC gradient (for gel band samples). Each sample was analyzed once for protein/peptide identification in data-dependent MS/MS mode and once for peptide isotope analysis in MS mode. Acquired MS/MS spectra were extracted and searched using Spectrum Mill Proteomics Workbench software (version B.04.00, Agilent Technologies) and either a rat or human protein database (http://www.uniprot.org/, release 2013_05). Search results were validated with a global false discovery rate of $1 \%$. A filtered list of peptides was collapsed into a nonredundant peptide formula database containing peptide elemental composition, mass, and retention time. This was used to extract mass isotope abundances (M0-M3) of each peptide from MS-only acquisition files with Mass Hunter Qualitative Analysis software (version B.05.00, Agilent Technologies). Software was developed at KineMed Inc. to calculate peptide elemental composition and curve-fit parameters for predicting peptide isotope enrichment (EMO) based on precursor body water enrichment ( $\mathrm{p}$ ) and the number ( $\mathrm{n}$ ) of amino acid $\mathrm{C}-\mathrm{H}$ positions per peptide actively incorporating hydrogen $(\mathrm{H})$ and deuterium $(\mathrm{D})$ from body water. Subsequent data handling was performed using Microsoft Excel templates, with input of precursor body water enrichment for each subject, to yield FSR data at the protein level. FSR data were filtered to exclude protein measurements with fewer than 2 peptide isotope measurements per protein. Details of FSR calculations and data filtering criteria were described previously $(30,31)$.

Statistics. Two-way ANOVA with post-hoc Holm-Sidak correction for multiple comparisons (GraphPad Prism) were performed to determine the differences between the groups for the rat denervation and clenbuterol study protein FSRs, as well as CK-M and CA-3 FSRs in muscle and plasma from the clenbuterol time-course study. Differences were considered significant at corrected $P<0.05$. Pearson correlation analysis (GraphPad Prism) was performed to correlate the $\mathrm{f}$ of CK-M and CA-3 in the muscle to that measured in the muscle. In addition, correlation analysis was also performed with $f$ of plasma CK-M and CA-3 with that of several myofibril and cytosolic proteins measured in the muscle. Correlations were considered significant at $P<0.05$. Heatmaps, hierarchal clustering, PCA analysis, and Pearson cross-correlation analysis of muscle protein FSR data were performed using Inferno ${ }^{1.0 b}$ for Proteomics. An in-house data analysis tool was developed to query and statistically analyze ontology terms of human muscle proteins, accessed programmatically from the DAVID v6.7. Gene ontology terms were retrieved based on an initial search of the proteins identified in the experimental datasets. The resulting terms were organized by categories (biological process, cellular component, and molecular function) and by levels (1-5). Duplicate terms within categories occurring at multiple levels were filtered to include only unique ontology terms at the lowest level. The median, standard deviation, and number of matching proteins were calculated for each 
ontology term, based on the corresponding proteins from experimental data. Paired 2-tailed $t$ tests with Benjamini-Hochberg multiple test corrections were then used to determine which terms were significantly enriched between experimental groups (corrected $P<0.05$ ). Significant terms were then further filtered by calculating the intersection of matching proteins within each ontology term in the same category and level, removing those terms with a minimum $80 \%$ intersection with other term(s) and retaining terms with the highest number of proteins. In addition, a between-group, unpaired 2-tailed $t$ test was also performed on 67 human muscle protein FSRs measured in all subjects; differences were considered significant with a false discovery rate of 0.2 after a Benjamini-Hochberg procedure was performed to adjust for the multiple comparisons.

Study approval. All animal studies were carried out according to NIH guidelines for the care and use of laboratory animals and received prior approval by the KineMed Animal Care and Use Committee. All human procedures and protocols described in the clinical studies were approved by the Institutional Review Boards at either Colorado State University (17 subjects: 7 males, 10 females) or BioMed IRB (San Diego, California, USA) (4 males). Each volunteer was informed of the potential risks and benefits and provided written consent before participating. The study followed the guidelines set forth by the Declaration of Helsinki.

\section{Author contributions}

MS, SMT, CB, KLH, BFM, and MKH designed the studies. CLK performed studies and sample analyses. MS, TEA, WEH, KWL, MC, and JCP developed methods. MS, TEA, WEH, KWL, MC, JCP, and MKH analyzed data. MS, CLK, WEH, TEA, and MKH wrote the manuscript.

\section{Acknowledgments}

We thank Joan Protasio, Chancy Fessler, Timothy Riff, and Corelle Nakamura for technical assistance. We thank a JCI reviewer for the suggestion about muscle mitochondrial-specific proteins as a potential future application of this approach.

The Office of Naval Research (N00014-10-1-0247 to C. Bell, K.L. Hamilton, and B.F. Miller) and KineMed Inc. supported this work.

Address correspondence to: Mahalakshmi Shankaran, KineMed Inc., 5980 Horton Street, Suite 470, Emeryville, California 94608, USA. Phone: 510.655.6525, ext. 133; E-mail: mshankaran@ kinemed.com.

John C. Price's present address is: Brigham Young University, Provo, Utah, USA.
1. Cawthon PM, et al. Do muscle mass, muscle density, strength, and physical function similarly influence risk of hospitalization in older adults? J Am Geriatr Soc. 2009;57(8):1411-1419.

2. Hairi NN, et al. Loss of muscle strength, mass (sarcopenia), and quality (specific force) and its relationship with functional limitation and physical disability: the Concord Health and Ageing in Men Project. JAm Geriatr Soc. 2010;58(11):2055-2062.

3. Dodson S, et al. Muscle wasting in cancer cachexia: clinical implications, diagnosis, and emerging treatment strategies. Annu Rev Med. 2011;62:265-279.

4. Lenk K, Schuler G, Adams V. Skeletal muscle wasting in cachexia and sarcopenia: molecular pathophysiology and impact of exercise training. J Cachexia Sarcopenia Muscle. 2010;1(1):9-21.

5. Bhasin S, et al. Older men are as responsive as young men to the anabolic effects of graded doses of testosterone on the skeletal muscle. JClin Endocrinol Metab. 2005;90(2):678-688.

6. Biolo G, Maggi SP, Williams BD, Tipton KD, Wolfe RR. Increased rates of muscle protein turnover and amino acid transport after resistance exercise in humans. Am J Physiol. 1995;268(3 pt 1):E514-E520.

7. Chesley A, MacDougall JD, Tarnopolsky MA, Atkinson SA, Smith K. Changes in human muscle protein synthesis after resistance exercise. JAppl Physiol (1985). 1992;73(4):1383-1388.

8. Ferrando AA, Tipton KD, Doyle D, Phillips SM, Cortiella J, Wolfe RR. Testosterone injection stimulates net protein synthesis but not tissue amino acid transport. Am J Physiol. 1998;275 (5 pt 1):E864-E871.

9. Sheffield-Moore M, et al. A randomized pilot study of monthly cycled testosterone replace- ment or continuous testosterone replacement versus placebo in older men. J Clin Endocrinol Metab. 2011;96(11):E1831-E1837.

10. Urban RJ, et al. Testosterone administration to elderly men increases skeletal muscle strength and protein synthesis. Am J Physiol. 1995;269 (5 pt 1):E820-E826.

11. Shankaran M, et al. Dynamic proteomics: a platform for proteome-wide interrogation of skeletal muscle anabolic response and non-invasive plasma biomarker discovery in humans and animal models. Presented at: New Directions in Biology and Disease of Skeletal Muscle Conference. Sixth Biennial Conference; June 29-July 2, 2014; Chicago, Illinois, USA. Abstract 96.

12. de Blaauw I, Deutz NE, von Meyenfeldt MF. Muscle protein and amino acid turnover in rats in vivo: effects of short-term and prolonged starvation. Clin Sci. 1996;90(6):457-466.

13. Hasten DL, Morris GS, Ramanadham S, Yarasheski KE. Isolation of human skeletal muscle myosin heavy chain and actin for measurement of fractional synthesis rates. Am J Physiol. 1998;275(6 pt 1):E1092-E1099.

14. Miller BF, et al. Coordinated collagen and muscle protein synthesis in human patella tendon and quadriceps muscle after exercise. J Physiol. 2005;567(pt 3):1021-1033.

15. Moore DR, Phillips SM, Babraj JA, Smith K, Rennie MJ. Myofibrillar and collagen protein synthesis in human skeletal muscle in young men after maximal shortening and lengthening contractions. Am J Physiol Endocrinol Metab. 2005;288(6):E1153-E1159.

16. Patterson BW, Zhang XJ, Chen Y, Klein S, Wolfe RR. Measurement of very low stable isotope enrichments by gas chromatography/ mass spectrometry: application to measure- ment of muscle protein synthesis. Metabolism. 1997;46(8):943-948.

17. Paddon-Jones D, Sheffield-Moore M, Aarsland A, Wolfe RR, Ferrando AA. Exogenous amino acids stimulate human muscle anabolism without interfering with the response to mixed meal ingestion. Am J Physiol Endocrinol Metab. 2005;288(4):E761-E767.

18. Tang JE, Moore DR, Kujbida GW, Tarnopolsky MA, Phillips SM. Ingestion of whey hydrolysate, casein, or soy protein isolate: effects on mixed muscle protein synthesis at rest and following resistance exercise in young men. J Appl Physiol (1985). 2009;107(3):987-992.

19. Bardocz S, Brown DS, Grant G, Pusztai A, Stewart JC, Palmer RM. Effect of the $\beta$-adrenoceptor agonist clenbuterol and phytohaemagglutinin on growth, protein synthesis and polyamine metabolism of tissues of the rat. Br J Pharmacol. 1992;106(2):476-482.

20. Maltin CA, Hay SM, Delday MI, Lobley GE, Reeds PJ. The action of the beta-agonist clenbuterol on protein metabolism in innervated and denervated phasic muscles. Biochem J. 1989;261(3):965-971.

21. Busch R, et al. Measurement of protein turnover rates by heavy water labeling of nonessential amino acids. Biochim Biophys Acta. 2006;1760(5):730-744.

22. Papageorgopoulos $\mathrm{C}$, Caldwell K, Schweingrubber H, Neese RA, Shackleton $\mathrm{CH}$, Hellerstein M. Measuring synthesis rates of muscle creatine kinase and myosin with stable isotopes and mass spectrometry. Anal Biochem. 2002;309(1):1-10.

23. Papageorgopoulos C, Caldwell K, Shackleton $\mathrm{C}$, Schweingrubber H, Hellerstein MK. Measuring protein synthesis by mass isotopomer 
distribution analysis (MIDA). Anal Biochem. 1999;267(1):1-16.

24. Robinson MM, Turner SM, Hellerstein MK, Hamilton KL, Miller BF. Long-term synthesis rates of skeletal muscle DNA and protein are higher during aerobic training in older humans than in sedentary young subjects but are not altered by protein supplementation. FASEB J. 2011;25(9):3240-3249.

25. Scalzo RL, et al. Greater muscle protein synthesis and mitochondrial biogenesis in males compared with females during sprint interval training. FASEB J. 2014;28(6):2705-2714.

26. Belloto E, Diraison F, Basset A, Allain G, Abdallah P, Beylot M. Determination of protein replacement rates by deuterated water: validation of underlying assumptions. Am J Physiol Endocrinol Metab. 2007;292(5):E1340-E1347.

27. Gasier HG, Fluckey JD, Previs SF. The application of $2 \mathrm{H} 2 \mathrm{O}$ to measure skeletal muscle protein synthesis. Nutr Metab (Lond). 2010;7:31.

28. Wilkinson DJ, et al. A validation of the application of $\mathrm{D}(2) \mathrm{O}$ stable isotope tracer techniques for monitoring day-to-day changes in muscle protein subfraction synthesis in humans. Am J Physiol Endocrinol Metab. 2014;306(5):E571-E579.

29. Decaris ML, et al. Proteomic analysis of altered extracellular matrix turnover in bleomycininduced pulmonary fibrosis. Mol Cell Proteomics. 2014;13(7):1741-1752.

30. Price JC, et al. Measurement of human plasma proteome dynamics with (2) $\mathrm{H}(2) \mathrm{O}$ and liquid chromatography tandem mass spectrometry. Anal Biochem. 2012;420(1):73-83.

31. Price JC, et al. The effect of long term calorie restriction on in vivo hepatic proteostatis: a novel combination of dynamic and quantitative proteomics. Mol Cell Proteomics. 2012;11(12):1801-1814.

32. Kasumov T, et al. Assessment of cardiac proteome dynamics with heavy water: slower protein synthesis rates in interfibrillar than subsarcolemmal mitochondria. Am J Physiol Heart Circ Physiol. 2013;304(9):H1201-H1214.

33. Lam MP, et al. Protein kinetic signatures of the remodeling heart following isoproterenol stimulation. J Clin Invest. 2014;124(4):1734-1744.

34. Clark RV, et al. Total body skeletal muscle mass: estimation by creatine (methyl-d3) dilution in humans. J Appl Physiol (1985). 2014;116(12):1605-1613

35. Stimpson SA, et al. Longitudinal changes in total body creatine pool size skeletal muscle mass using the $\mathrm{D}$-creatine dilution method [published online ahead of print June 25, 2013]. JCachexia Sarcopenia Muscle. doi:10.1007/ s13539-013-0110-1.

36. Stimpson SA, et al. Total-body creatine pool size and skeletal muscle mass determination by creatine-(methyl-D3) dilution in rats. J Appl Physiol (1985). 2012;112(11):1940-1948.

37. Goldspink DF. The effects of denervation on protein turnover of rat skeletal muscle. Biochem J. 1976;156(1):71-80.

38. Urdal P, Urdal K, Stromme JH. Cytoplasmic creatine kinase isoenzymes quantitated in tissue specimens obtained at surgery. Clin Chem. 1983;29(2):310-313.
39. Wu AH, Perryman MB. Clinical applications of muscle enzymes and proteins. Curr Opin Rheumatol. 1992;4(6):815-820.

40. Jeffery S, Edwards Y, Carter N. Distribution of CAIII in fetal and adult human tissue. Biochem Genet. 1980;18(9-10):843-849.

41. Kato K, Mokuno K. Distribution of immunoreactive carbonic anhydrase III in various human tissues determined by a sensitive enzyme immunoassay method. Clin Chim Acta. 1984;141(2-3):169-177.

42. Wistrand PJ. Carbonic anhydrase III in liver and muscle of male rats purification and properties. Ups J Med Sci. 2002;107(2):77-88.

43. Young VR. Regulation of protein synthesis and skeletal muscle growth. J Anim Sci. 1974;38(5):1054-1070.

44. Huang da W, Sherman BT, Lempicki RA. Systematic and integrative analysis of large gene lists using DAVID bioinformatics resources. Nat Protoc. 2009;4(1):44-57.

45. Huang da W, Sherman BT, Lempicki RA. Bioinformatics enrichment tools: paths toward the comprehensive functional analysis of large gene lists. Nucleic Acids Res. 2009;37(1):1-13.

46. Apple FS, Rogers MA, Ivy JL. Creatine kinase isoenzyme MM variants in skeletal muscle and plasma from marathon runners. Clin Chem. 1986;32(1 pt 1):41-44.

47. Pagliarini DJ, et al. A mitochondrial protein compendium elucidates complex I disease biology. Cell. 2008;134(1):112-123.

48. Calvo SE, Clauser KR, Mootha VK. MitoCarta2.0: an updated inventory of mammalian mitochondrial proteins [published online ahead of print October 7, 2015]. Nucleic Acids Res. doi:10.1093/nar/gkv1003.

49. Decaris ML, et al. Turnover rates of hepatic collagen and circulating collagen-associated proteins in humans with chronic liver disease. PLoS One. 2015;10(4):e0123311.

50. Bateman RJ, Munsell LY, Chen X, Holtzman DM, Yarasheski KE. Stable isotope labeling tandem mass spectrometry (SILT) to quantify protein production and clearance rates. J Am Soc Mass Spectrom. 2007;18(6):997-1006

51. Kasumov T, et al. Measuring protein synthesis using metabolic (2)H labeling, high-resolution mass spectrometry, and an algorithm. Anal Biochem. 2011;412(1):47-55.

52. Lee AY, et al. Measurement of fractional synthetic rates of multiple protein analytes by triple quadrupole mass spectrometry. Clin Chem. 2012;58(3):619-627.

53. Shankaran M, et al. Dynamic proteomics approach using heavy water $(2 \mathrm{H} 2 \mathrm{O})$ labeling to measure turnover rates of proteins secreted by adult rat hippocampal stem / progenitor cells. Presented at: Society for Neuroscience Annual Meeting; November 14, 2011; Washington, DC, USA. Program 518.22/ZZ47.

54. Shankaran M, et al. Measurement of proteome dynamics in brain cerebrospinal fluid (CSF) by heavy water labeling: Lipopolysaccharideinduced neuro-inflammation, treatment with a FMS kinase inhibitor translation into human CSF. Presented at: Society for Neuroscience Annual Meeting; November 13, 2013; San Diego,
California, USA. Program 725.09/U8.

55. Geiger $\mathrm{T}$, et al. Initial quantitative proteomic map of 28 mouse tissues using the SILAC mouse. Mol Cell Proteomics. 2013;12(6):1709-1722.

56. Erve JC, Gu M, Wang Y, DeMaio W, Talaat RE. Spectral accuracy of molecular ions in an LTQ/ Orbitrap mass spectrometer and implications for elemental composition determination. J Am Soc Mass Spectrom. 2009;20(11):2058-2069.

57. Maltin CA, Hay SM, Delday MI, Smith FG, Lobley GE, Reeds PJ. Clenbuterol, a $\beta$ agonist, induces growth in innervated and denervated rat soleus muscle via apparently different mechanisms. Biosci Rep. 1987;7(6):525-532.

58. Sato Y, et al. Differential expression of sarcoplasmic and myofibrillar proteins of rat soleus muscle during denervation atrophy. Biosci Biotechnol Biochem. 2009;73(8):1748-1756.

59. Sun H, Li M, Gong L, Liu M, Ding F, Gu X. iTRAQcoupled 2D LC-MS/MS analysis on differentially expressed proteins in denervated tibialis anterior muscle of Rattus norvegicus. Mol Cell Biochem. 2012;364(1-2):193-207.

60. Sun H, Liu J, Ding F, Wang X, Liu M, Gu X. Investigation of differentially expressed proteins in rat gastrocnemius muscle during denervation-reinnervation. J Muscle Res Cell Motil. 2006;27(3-4):241-250.

61. Batt J, et al. Differential gene expression profiling of short and long term denervated muscle. FASEB J. 2006;20(1):115-117.

62. Milot J, Fremont P, Cote C, Tremblay RR. Differential modulation of carbonic anhydrase (CA III) in slow- and fast-twitch skeletal muscles of rat following denervation and reinnervation. Biochem Cell Biol. 1991;69(10-11):702-710.

63. Wistrand PJ, Carter ND, Askmark H. Induction of rat muscle carbonic anhydrase by denervation demonstrated with immunofluorescence. Comp Biochem Physiol A Comp Physiol. 1987;86(1):177-184.

64. Burgomaster KA, Hughes SC, Heigenhauser GJ, Bradwell SN, Gibala MJ. Six sessions of sprint interval training increases muscle oxidative potential and cycle endurance capacity in humans. J Appl Physiol (1985). 2005;98(6):1985-1990.

65. MacDougall JD, Hicks AL, MacDonald JR, McKelvie RS, Green HJ, Smith KM. Muscle performance and enzymatic adaptations to sprint interval training. J Appl Physiol (1985). 1998;84(6):2138-2142.

66. Burgomaster KA, Heigenhauser GJ, Gibala MJ. Effect of short-term sprint interval training on human skeletal muscle carbohydrate metabolism during exercise and time-trial performance. J Appl Physiol (1985). 2006;100(6):2041-2047.

67. Parra J, Cadefau JA, Rodas G, Amigo N, Cusso R. The distribution of rest periods affects performance and adaptations of energy metabolism induced by high-intensity training in human muscle. Acta Physiol Scand. 2000;169(2):157-165.

68. McArdle A, Pattwell D, Vasilaki A, Griffiths RD, Jackson MJ. Contractile activity-induced oxidative stress: cellular origin and adaptive responses. Am J Physiol Cell Physiol. 2001;280(3):C621-C627.

69. Morton JP, et al. Reduced carbohydrate availability 
does not modulate training-induced heat shock protein adaptations but does upregulate oxidative enzyme activity in human skeletal muscle. J Appl Physiol (1985). 2009;106(5):1513-1521.

70. Mori M, Higuchi K, Sakurai A, Tabara Y, Miki T, Nose H. Genetic basis of inter-individual variability in the effects of exercise on the alleviation of lifestyle-related diseases. J Physiol. 2009;587(pt 23):5577-5584.

71. Uhlen M, et al. Proteomics. Tissue-based map of the human proteome. Science. 2015;347(6220):1260419.

72. Shankaran M, et al. Muscle protein fractional synthesis rates predicts muscle mass gain in response to a selective androgen receptor modulator. J Frailty Aging. 2015;4(suppl 1):38.
73. Hellerstein MK, Murphy E. Stable isotope-mass spectrometric measurements of molecular fluxes in vivo: emerging applications in drug development. Curr Opin Mol Ther. 2004;6(3):249-264.

74. Beehler BC, Sleph PG, Benmassaoud L, Grover GJ Reduction of skeletal muscle atrophy by a proteasome inhibitor in a rat model of denervation. Exp Biol Med (Maywood). 2006;231(3):335-341. 